\title{
Cross-layered resource allocation in UWB noise-OFDM-based ad hoc surveillance networks
}

\author{
Shrawan Chittoor Surender ${ }^{1}$, Ram M Narayanan ${ }^{2 *}$ and Chita R Das ${ }^{1}$
}

\begin{abstract}
Situational awareness in military surveillance and emergency responder scenarios requires detection of long range targets and secure communication of this information across a multi-sensor network. A potential approach towards this requirement is to harness the coexisting advantages of radar sensing and wireless communications. A multifunctional communications-embedded radar design that implicitly develops into a cross-layered multi-radar secure wireless ad hoc network is proposed to address this need. First, we demonstrate radar and communications coexistence through analysis of our novel composite orthogonal frequency division multiplexing (OFDM)-embedded ultra wideband (UWB) noise waveform's bit error rate and ambiguity function formulations. Second, to solve the medium access problem of allocating the multiple OFDM frequencies between different ad hoc radars nodes, we propose a simple yet fully distributed, channel-diversity-aided algorithm. It constructs a contention-free network, scaling logarithmically with the number of radar nodes, and analytically guarantees a provable fraction of the maximum throughput achieved by any optimal centralized allocation algorithm. Furthermore, our solution dynamically adapts with channel variations and topology changes. Working in-sync with the UWB noise-based single radio multi-channel wireless platform, this distributed resource allocation builds a synergistic cross-layered ad hoc network of radars.
\end{abstract}

Keywords: Coexistence, Radar-communications, UWB, Noise-OFDM, Multi-radar, Ad hoc networks, Cross-layer, Surveillance networks, Distributed resource allocation

\section{Introduction}

Recently, the wireless networks research community has taken considerable interest in concurrently utilizing the ultra wideband (UWB) spectrum with narrow-band systems to architect coexisting and cognitive spectrum solutions $[1,2]$. In parallel, multi-channel multi-radio wireless systems are being developed to achieve improved network capacity by exploiting non-overlapping channel transmissions [3]. In this study, our motivation is to exploit the synergy between the above two approaches, in developing a multi-functional wireless system with significant support to applications of practical relevance. The goal is to provide a flexible framework for situational awareness in infrastructure-less covert surveillance circumstances,

\footnotetext{
*Correspondence: ram@engr.psu.edu

2 Department of Electrical Engineering, The Pennsylvania State University, University Park, PA 16802, USA

Full list of author information is available at the end of the article
}

such as hostage rescue, first-emergency response, natural disaster recovery, or military surveillance. The primary requirements for such applications are the capability for far-field sensing of phenomena, distributed computing, and secure multi-sensor networking. These situations are also characterized by the absence of any central infrastructure, limited spectrum availability, varying topological environments, and growing wireless security concerns [4].

A typical application scenario is shown in Figure 1, where a set of first-emergency responders (FERs) are surrounding a building under siege with hostages inside. Accurate situational awareness is of invaluable help here. If each FER carries a radar sensor, he/she can individually detect unknown targets. However, collective data from each of the FERs, if processed together, can transform these localized individual detections to highly accurate situational awareness. Towards achieving this objective, the individual radar sensors must form a wireless communication platform among themselves.

\section{是 Springer}

(c) 2013 Surender et al: licensee Springer. This is an Open Access article distributed under the terms of the Creative Commons Attribution License (http://creativecommons.org/licenses/by/2.0), which permits unrestricted use, distribution, and reproduction in any medium, provided the original work is properly cited. 


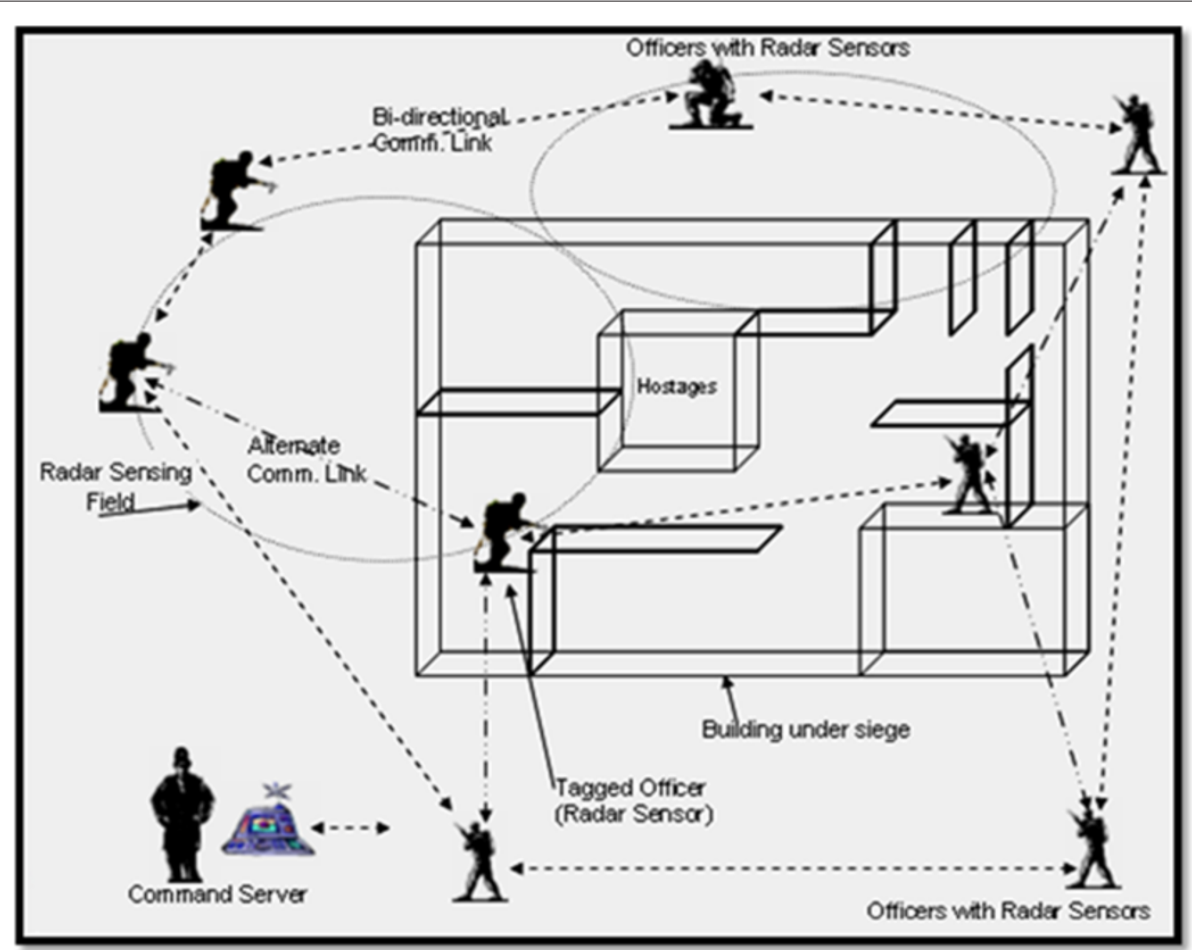

Figure 1 First-emergency hostage rescue operational scenario with multiple radar sensors.

An ad hoc network harnessing the combined advantages of UWB radar-sensing $[5,6]$ and wireless communications is a viable solution to this requirement. This is accomplished through a unique multi-functional radar design, where we spectrally notch a UWB radar's random noise transmit waveform and embed a secure spectrally equalized orthogonal frequency division multiplexed (OFDM) multi-carrier message within the UWB spectrum. Thus, the composite transmit waveform is designed to simultaneously support far-field target-sensing and data communications, while possessing covertness and interference rejection properties owing to the transmission of a noise-like signal. This single-radio multi-channel wireless radar-communications system is what we call the UWB noise-OFDM radar [7]. Multiple such radars can then form an ad hoc communication network to provide significant benefits in target detection and recognition, by taking advantage of multi-aspect and multi-look fusion techniques $[8,9]$, and information dissemination through opportunistic routing [10]. Furthermore, by having multicarrier data communication within the same spectrum as the radar's transmit signal, a substantial increase in network capacity and bandwidth efficiency can be achieved. This article, therefore, aims to transform a multi-functional UWB noise-OFDM radar into a coexisting, cross-layered, covert, and ad hoc network platform.

The two important parameters investigated for performance analysis are the bit error rate (BER) reliability measure for communications and the ambiguity function $(\mathrm{AF})$ resolution measure for radar operations [11]. Furthermore, the presence of the OFDM-based multicarrier PHY and the absence of any coordinating central infrastructure necessitated access control to be exercised on the wireless medium in a distributed and efficient manner. Based on the available OFDM bandwidth and the modulation parameters, there exists a set number of orthogonal subcarriers available to each radar. All communications between radars are required to be channeled through these subcarriers only. This article presents a fully distributed contention-free medium access strategy that simultaneously exploits channel diversity and maximizes data reliability. This strategy is also scalable and adaptable to changing network topologies and varying wireless channels. This problem is similar to the multiradio multi-channel resource allocation problem considered in $[3,12,13]$; however, it is further constrained by the requirements of multi-functionality and covertness. In short, the key contributions of this study are as follows:

- Through experimental simulations, we show that this radar-embedded multi-user communication platform achieves a BER value better than $1 \times 10^{-5}$ for an signal-to-noise ratio (SNR) of $12 \mathrm{~dB}$, thus forming a reliable physical layer for the wireless network of radars. 
- By formulating and deriving the $\mathrm{AF}$ for the radar part of the noise-OFDM radar, we infer that if the fragmented spectral gap is less than $30 \%$, then range and Doppler resolution, i.e., the radar performance, are essentially unaffected.

- To have contention-free medium access of this $30 \%$ available OFDM bandwidth among multiple radars, we propose a fully distributed Channel-diversityaided Propose-Accept-Reject-Confirm (CPARC) resource allocation algorithm. Through simulations, CPARC is shown to scale logarithmically with the number of radars, and to adapt automatically to channel and topology variations.

- We analytically prove that our resource allocation strategy guarantees a constant fraction of the maximum throughput region offered by any optimal centralized offline algorithm.

- Finally, we highlight the improved performance of CPARC when compared with other related algorithms in the literature (centralized edge-coloring algorithm and distributed frequency-hopping OFDM technique).

The remainder of this article is organized as follows. Section 2 presents related work. System design of our secure wireless platform is briefly described in Section 3. In Section 4, we show its physical layer coexistence capability by investigating the performance of the communication and radar operations. To combine the individual radars into a multi-radar multi-hop ad hoc communication network, Section 5 formulates the ad hoc multi-carrier resource allocation problem, while Section 6 proposes a fully distributed channel-sensing medium access solution. The throughput guarantee of our system is analyzed in Section 7. In Section 8, extensive numerical simulations are presented to validate the radarcommunications coexisting spectrum access performance and to evaluate the expected performance of CPARC. We give our concluding comments in Section 9.

\section{Related work}

Compared to generic multi-static or netted radar systems [14], the UWB noise-OFDM radar is a distinct coexisting solution where the same transmit signal is simultaneously used for both radar and communication operations, and it is inherently secure. In [15], a comparable dual-system is presented which uses UWB OFDM pulses for radar and communication. While this is indeed a viable alternative, immunity from detection requires random frequency hopping. Furthermore, the UWB OFDM pulses show greater range side lobes compared to Gaussian pulses and the communication system requires full synchronization between the platforms. Further examining the existing UWB radar-communication systems, we observed that they are either not multi-radar capable [16] or are easily jammed open communication systems [15]. In contrast, we present a multi-functional UWB netted radar with better coexistence of radar and communication abilities, along with inherent transmit waveform covertness and multi-radar capability.

The current medium access protocols proposed for narrow-band wireless systems such as carrier sense multiple access (CSMA), IEEE 802.11, IEEE 802.15.3, etc., are unsuitable for UWB systems such as ours, for a variety of reasons [17]. For instance, energy-based clear channel assessment is necessary in CSMA/CA, however, UWB is defined to have low-energy emissions. Furthermore, synchronization signaling overhead is very high in the above protocols, which UWB systems cannot handle. Next, among the solutions proposed for OFDM-based dynamic subcarrier allocation $[18,19]$, almost all of these techniques use a central coordinator or hierarchical coordinators to run the optimal/suboptimal subcarrier allocation algorithm and then flush the subcarrier allocations to all users (receivers) through a separate control channel. These typical downlink-based cellular-type solutions [19] cannot, therefore, be applied to a system where there is no centralized infrastructure. However, [20] does present an interesting distributed spectrum access technique for OFDM systems.

Popular techniques in multi-hop distributed scheduling $[13,21]$ come close to satisfying our requirements. However, the approach presented in [21] is meant only for single-channel systems. Lin and Rasool's study [13] proposes a multi-channel joint scheduling and channelassignment algorithm. It mainly depends on the channel queue length information being exchanged between interfering links, but it is assumed to be available to the distributed algorithm. In contrast, our strategy relaxes this assumption and includes channel diversity-based assignments, thereby, achieving a marginally better throughput guarantee than [13]. Dutta et al's study [12] solves the channel assignment problem, but it does not consider dynamic network topologies or channel variations and hence does not exploit channel diversity. Finally, a recent multi-radio multi-channel resource allocation based on a multi-dimensional conflict graph technique [3] is a novel approach, but it cannot be inherently applied to an ad hoc network. In contrast, our approach presented in Section 5 is a fully distributed channel-aware solution appropriate for an ad hoc distributed computing environment.

\section{Background: secure wireless platform design}

The UWB noise-OFDM netted radar (in short, radar sensor) system architecture, performing: (1) radar-specific operations such as target detection, ranging, velocity (Doppler) estimation, and tracking, and (2) datanetworking with other radar sensors and the command 
center for distributed computing, consists of transmit and receive subsystems as summarized below.

\subsection{Transmit subsystem}

As seen in Figure 2, a noise generator produces a signal with a Gaussian amplitude distribution and approximately constant power spectral density (PSD). This signal is filtered in a band-pass filter (BPF) to generate a band-limited UWB noise signal within a range of $\left(f_{1}, f_{2}\right) \mathrm{Hz}$. Such a band-pass Gaussian process of bandwidth $\beta$ and centered at some frequency $f_{o}=\left(f_{1}+f_{2}\right) / 2$, where $f_{o}>\beta / 2$, can be expressed as

$$
X_{G}(t)=\frac{1}{2}\left[u(t) \exp \left(j 2 \pi f_{o} t\right)+u^{*}(t) \exp \left(-j 2 \pi f_{o} t\right)\right],
$$

where $u(t)=X_{c}+j X_{s}$ is the complex envelope and $*$ indicates the complex conjugation operation.

In our simulations, we used a $1-2-\mathrm{GHz}$ frequency range of operation. Since the original UWB radar, which is already operational, was built in the $1-2-\mathrm{GHz}$ range, we continued to develop this system in the same range. Changing to FCC allowable ranges is trivial and the design can easily be adapted to it. For our system therefore, $f_{o}=$ $1.5 \mathrm{GHz}, f_{1}=1 \mathrm{GHz}$, and $f_{2}=2 \mathrm{GHz}$. This is a UWB signal by definition since its fractional bandwidth $\left(\beta / f_{o}\right)$ is greater than 0.25 . This band-limited noise signal is then notch-filtered within the narrower range of $1.2-1.3 \mathrm{GHz}$ (in general, this could be done over any range suitable for the application). The radar system therefore uses the full 1-2 GHz band, while the intra-sensor communications operates over the $1.2-1.3 \mathrm{GHz}$ sub-band. The Gaussian process $X_{N}(t)$ in the notched band has bandwidth $\beta_{n}$ and is centered at frequency $f_{n}$, such that $f_{o}-\beta / 2<f_{n}<$ $f_{o}+\beta / 2$.

We use a random binary number generator to generate test data. In general, this would represent the target data collected by the radar, or any other information it wishes to communicate to other radars in its vicinity. Since an
OFDM waveform's spectral characteristics closely mimic that of white noise, we map this data onto OFDM subcarriers to generate the OFDM symbols, and thereby conceal the data within the noise. A pass-band real OFDM signal with a communication carrier $f_{n}$ can be represented as [22]

$$
X_{D}(t)=\frac{1}{2}\left[s(t) \exp \left(j 2 \pi f_{n} t\right)+s^{*}(t) \exp \left(-j 2 \pi f_{n} t\right)\right],
$$

where

$$
s(t)=\sum_{k=0}^{N-1} s_{k} \exp \left(j 2 \pi f_{k} t\right)=s_{I}(t)+j s_{Q}(t),
$$

with $s_{k}=d_{k} e^{j \theta_{k}}$ being the complex data symbols that are modulated onto the OFDM subcarriers and $f_{k}=f_{\mathrm{b}}+k \Delta f$. Here, $f_{\mathrm{b}}$ is the base frequency, $\Delta f$ is the subcarrier spacing, and $N$ is the total number of subcarriers. These parameters are appropriately selected so that the bandwidth of this OFDM signal exactly matches the notch bandwidth in $X_{N}(t)$.

The above OFDM signal is inserted within the notched band-limited noise signal, with appropriate power equalization and an ideal mixer, to produce the multi-functional noise-OFDM radar transmit waveform, which is propagated by an omni-directional transmit antenna. As seen by any unintended receiver, the transmitted composite waveform clearly appears random and noise-like [7], preserving its covertness properties and hence presenting physical layer security. However, for other friendly radar sensors in the transmitting radar's vicinity, the waveform is construed as data plus noise. We show four different power spectrum plots in Figure $3 \mathrm{a}-\mathrm{d}$ to demonstrate that the transmitted signal does appear noise-like to the outside observer. Note from Figure 3d for the OFDM embedded in notched UWB noise that the spectrum appears completely random and noise-like, similar to that shown in Figure $3 \mathrm{~b}$ for UWB random noise.

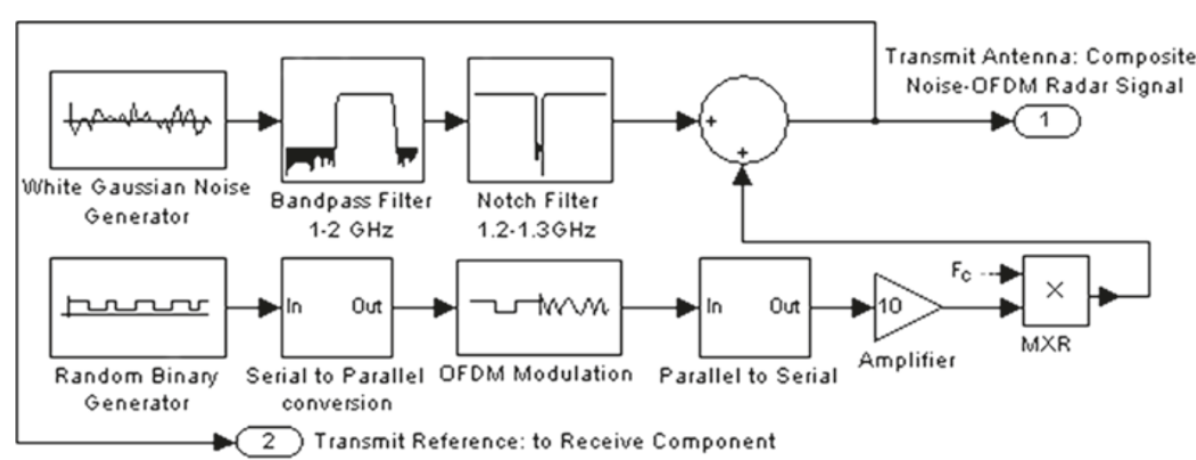

Figure 2 UWB noise-OFDM radar: transmit subsystem architecture. 

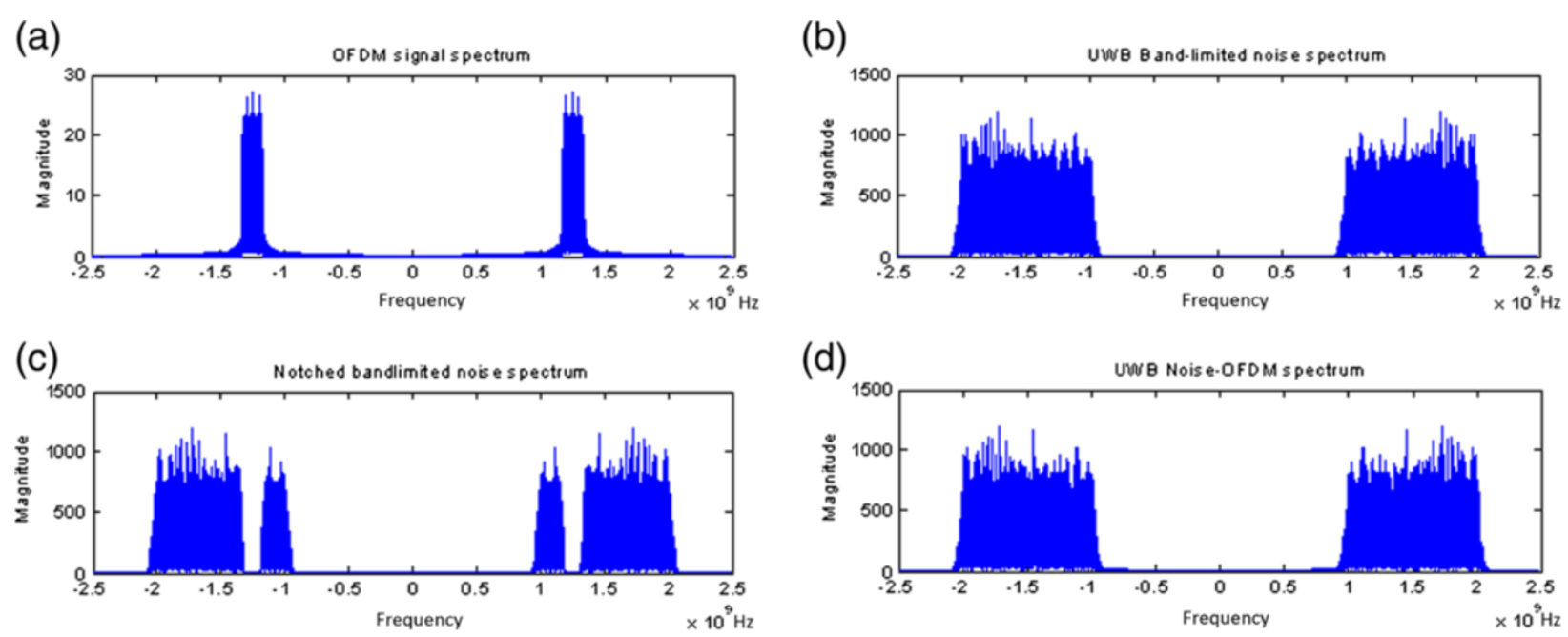

Figure 3 Spectrum comparison for covertness. Spectrum: (a) OFDM signal, (b) band-limited UWB noise, (c) UWB notched noise, and (d) Coexistence: UWB noise-OFDM spectrum. Comparing (b) and (d), we note that the transmit waveform (d) also appears random \& noise-like, thereby hiding the data message inside.

\subsection{Receive subsystem}

Due to the omni-directional nature of the transmit antenna and its relatively high transmit power, the noiseOFDM waveform is likely to be picked up by several radar sensors in the neighborhood of the transmitting radar. These radar sensors process this signal within their communication-receiver subsystem, shown in the top part of Figure 4. Here, the concealed digital data are retrieved following the steps of frame and timing detection, band-pass filtering the signal within the $1.2-1.3 \mathrm{GHz}$ range, followed by OFDM demodulation, decoding, and detection. The composite noise-OFDM transmit signal also travels to the target within the radar's range and returns as target backscatter. In the transmitting radar, this echo is processed by the radar-receiver subsystem, shown in the lower part of Figure 4. The reference transmit noise-OFDM waveform is passed through a delay line and fed into one input of an up-converter MXR1. A local oscillator at $f_{I F}$ also feeds into MXR1. The frequency shifted and time-delayed output of MXR1 is passed through correlator MXR2 along with the incoming target echo. The correlator maximizes the output SNR and the knowledge of the delay helps in determining the range to the target. Passing the output of MXR2

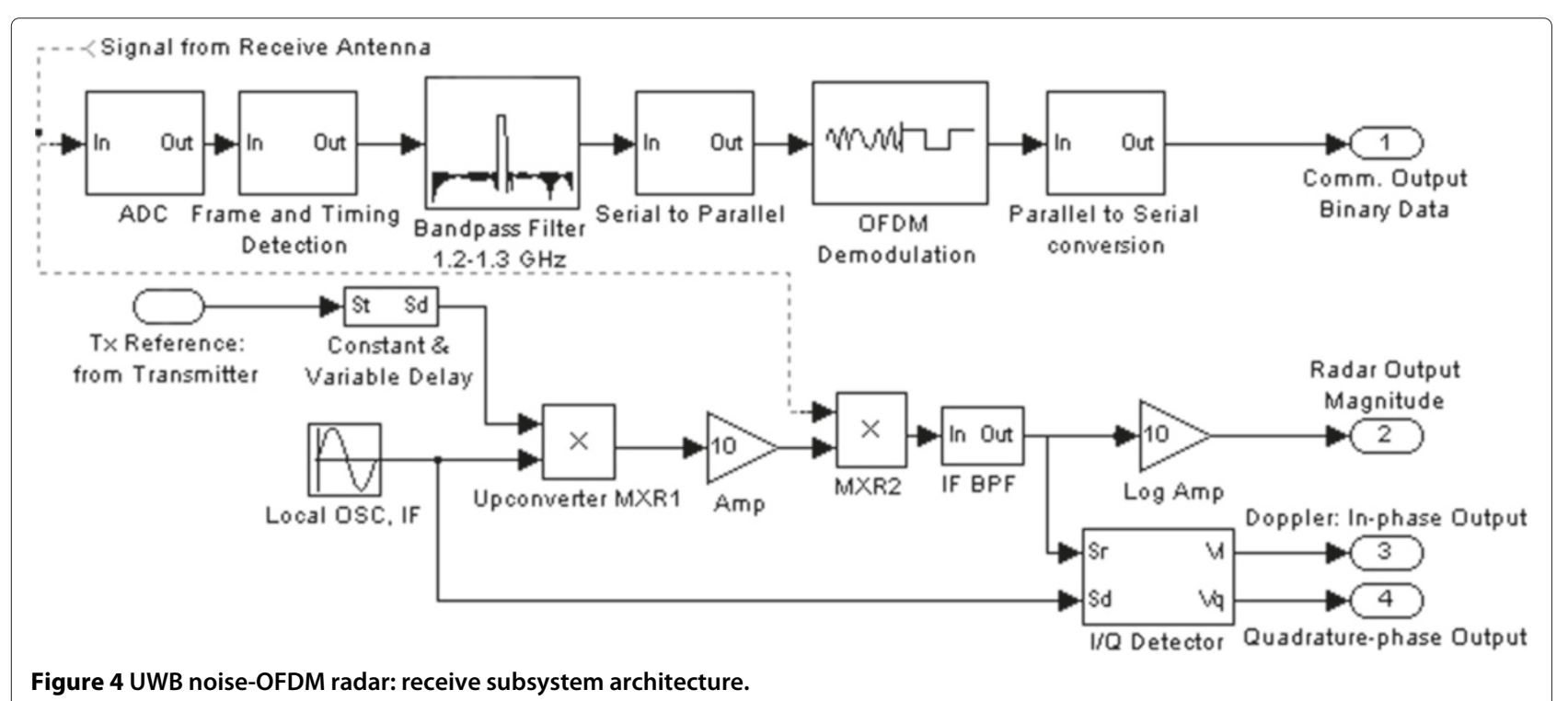


through an IF BPF, we get an estimate of the magnitude of the target reflectively. By feeding this signal through an in-phase/quadrature (I/Q) detector, Doppler information about the target can be obtained. In this study, we assume that carrier-frequency offsets and I/Q imbalance have been corrected at the OFDM demodulation side.

\section{Physical layer coexistence}

To establish coexistence of communications with radar transmissions, the receive subsystem must be analyzed further to address two essential issues: (1) effect on communications performance due to the presence of UWB noise around the OFDM signal, and (2) effect on the radar's range and Doppler estimation capabilities due to the presence of an OFDM spectrum embedded inside the UWB random noise transmit spectrum.

\subsection{Noise-embedded-communications performance}

BER is one of the most widely used metrics for gauging the reliability of a communication technique. For the noiseOFDM radar, reliability depends on various parameters: SNR at the input of the receiving antenna, number of data subcarriers used, power difference between the notched noise signal and OFDM signal, and BPF spectral characteristics. The SNR at the input of the receiving antenna is defined as the ratio of the transmitted noise-OFDM signal to the band-pass undesired channel noise received by the antenna. The general BER of the noise-OFDM radar's communication subsystem has been derived to be [7]

$$
P_{b}=\frac{1}{N} \sum_{l=-\frac{N}{2}}^{l=\frac{N}{2}} P_{b}\left(Y_{l}\right)=\frac{1}{N} \sum_{l=-\frac{N}{2}}^{l=\frac{N}{2}} Q\left(\sqrt{\frac{v_{l}^{2} \epsilon_{s, l}}{\sigma_{y, l}^{2}}}\right) .
$$

where $Y_{l}$ is the received signal on the $l$ th sub-carrier, $\epsilon_{s, l}$ is the signal energy of the symbol being modulated onto the $l$ th OFDM sub-carrier, $v_{l}$ is the FIR filter response's magnitude ripple coefficient affecting the $l$ th OFDM subcarrier, and $\sigma_{y, l}^{2}$ is the variance of the additive white Gaussian noise on the $l$ th OFDM sub-carrier. The model described by Equation (4) agrees with experimental simulations, as shown in [7]. We mention it here since it validates the multi-user BER versus SNR experimental simulations presented in Section 8.

\subsection{Target detection performance}

In this section, we examine the radar side of the coexisting system. The UWB noise radar has been shown to possess high range resolution and good velocity resolution capabilities [23]. Range resolution signifies the capability of the radar to distinguish between two closely separated targets. For a 1-GHz bandwidth UWB noise radar, targets separated by $15-\mathrm{cm}$ spacing in the range dimension will correctly be detected as two separate targets. Velocity resolution represents the radar's ability to estimate the speed of different moving targets whose velocities are closely separated. Since the transmit waveform of the noise radar was modified to embed a communication signal within, we investigate the UWB noise-OFDM radar's range and velocity resolution performance by formulating its AF.

For conventional radars, the transmit signal envelope is of a deterministic nature for which a matched filter can be realized, and the time-invariant AF formulation establishes its range and velocity resolution properties. However, for a random noise radar, a matched filter cannot be realized; instead a correlator matched to the transmit process is required. Since the transmit waveform output of a noise radar is a random function of time, only its statistics can be described. Therefore, an analogous AF may be defined as the expected value of the response of the correlator matched to a target at a desired range moving at a desired velocity, to the return signal from the target at a different range moving at a different velocity. To discuss the range and Doppler resolution issues pertaining to the UWB noise-OFDM radar, we derived its generalized wide-band AF (shown in Appendix) from first principles. The resulting expression for the AF of a UWB noise-OFDM radar [24] is

$$
|\langle\chi(\alpha, \tau, t)\rangle|=|(A+B) \otimes h(t)|,
$$

with

$$
A=R_{c c}(\alpha t-\tau) \exp \left(-j 2 \pi \alpha f_{o} t\right),
$$

and

$$
B=\left(R_{c c_{n}}(\alpha t-\tau)+N T_{s}\right) \exp \left(-j 2 \pi \alpha f_{n} t\right),
$$

where $h(t)$ is the integration filter impulse response, $\alpha$ is the delay rate associated with the target's velocity, $\tau(t)=$ $\tau_{o}-\alpha t$ is the delay associated with the target's range, and $R_{c c}, R_{c c_{n}}$ are the auto-correlation functions of the UWB noise and notched noise, respectively.

Our simulations of this AF, in Section 8, clearly demonstrate that the OFDM data bandwidth can be as high as $30 \%$ of the complete UWB bandwidth available to the radar, without adversely affecting ranging or Doppler estimation performance and the noise-like properties of the radar transmit waveform.

\section{Cross-layer problem formulation: multi-user resource allocation}

Based on this available OFDM data bandwidth and the selection of the OFDM modulation parameters, there is a set amount of orthogonal subcarriers available to every radar in the system. In our case, we chose $3.125 \mathrm{MHz}$ bandwidth for each subcarrier, thereby filling the $0.1 \mathrm{GHz}$ notch band with 32 subcarriers. Individual radars can now use these subcarriers to concurrently communicate with 
their neighboring radars, while simultaneously performing radar functions, such as target detection. However, when a number of radars are deployed and all radars transmit on all 32 subcarriers, collisions on the channel will inhibit effective communication within the intended network. The goal therefore is to achieve medium access, i.e., to allocate these subcarriers in such a manner as to establish contention-free communication links between every radar and its one-hop neighbors. We call this radar pairing, i.e., every radar needs to be paired with each of its neighboring radars on a different subcarrier. All communication between the radar pairs must occur through these assigned subcarriers only. Furthermore, the allocation of these subcarriers between the radar pairs must be accomplished under changing radar topologies and the following unique constraints of an infrastructure-less multi-radar setup:

- Multi-echo issue: a radar receives signals not only from a transmitting radar in the neighborhood, but also the target echoes due to its own transmissions as well as the echoes due to transmissions of other radars in the vicinity, as depicted in Figure 5. Here, RS1 and RS2 transmit out their UWB Noise-OFDM signals $\psi_{1}$ and $\psi_{2}$, respectively. RS2 being in the vicinity of RS1, receives $\psi_{1}$ and primarily needs to extract data from it, but RS2 also receives the target echoes, $\psi_{1 e}$ and $\psi_{2 e}$, which will lead to demodulation errors in the OFDM band. It is, therefore, important that the subcarriers, on which RS1 transmits data to RS2, are not affected by the corresponding subcarriers in the target echoes.
- Ad hoc network: it is a multi-hop network of radars, with no single central coordinator to crunch an optimal or sub-optimal resource allocation algorithm and disseminate the information to all radars in the system.

- Channel diversity: due to terrain obstruction and other wireless channel issues, some subcarriers might not inherently be suitable for pairing a particular set of radars. Making such an allocation might degrade the data reliability of the system. Therefore, channel estimation is needed in exploiting channel diversity.

The overall problem is to minimize the number of subcarriers used for communication under the above constraints, which is analogous to maximizing subcarrier reuse in the overall system.

A naive solution is to statically assign subcarriers in the OFDM spectrum to different radar transmitter-receiver pairs. However, this does not permit exploitation of the benefits accruing from channel and spatial diversities. This static allocation will lead to disconnected radars and under-utilization of the bandwidth spectrum especially when the radars are moving or when the radio channel changes.

\section{A simple distributed channel-diversity approach}

Our aim is to pair, i.e., to allocate OFDM subcarriers to different (source radar, destination radar) duple's dynamically, so that collision-free communication can occur simultaneously among the radars. A small subset of the available subcarriers in the OFDM spectrum is chosen

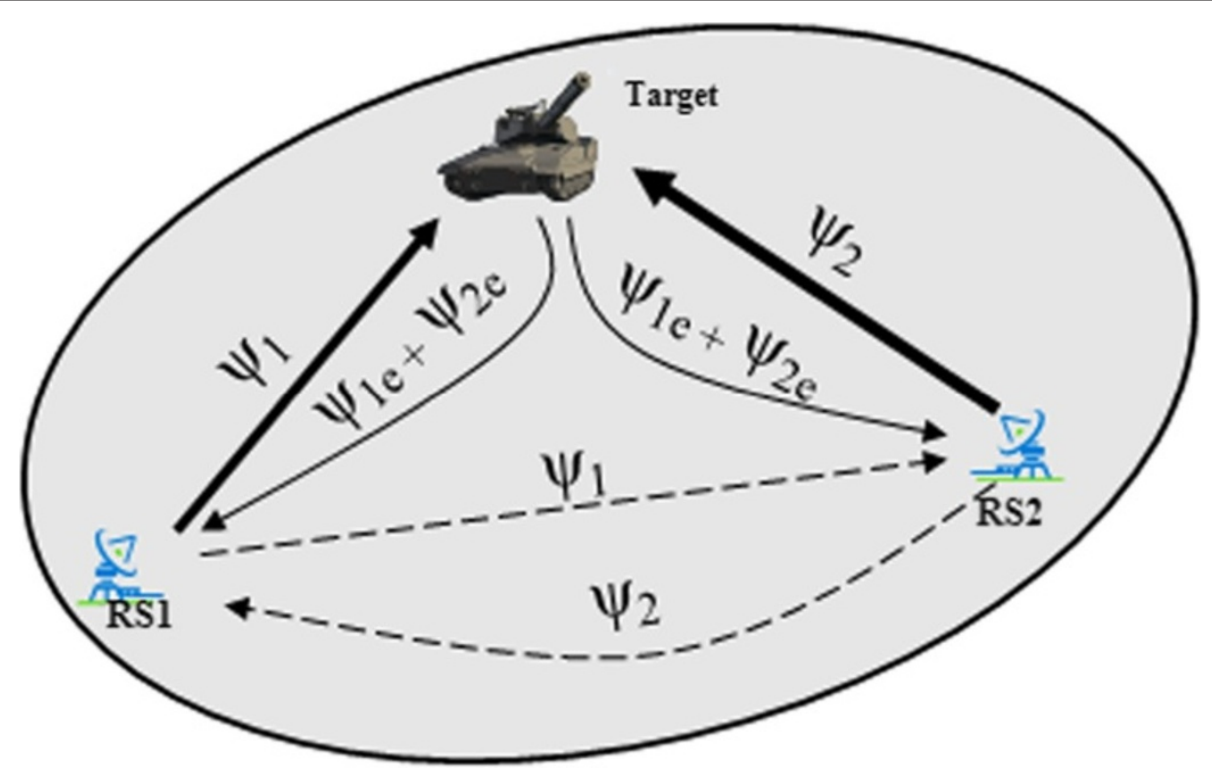

Figure 5 Multi-echo issue. 
as a control carrier set $(\mathrm{CC})$, which is used by all the radars in the system as a broadcast channel for information exchange. All radars in the system will contend to use this channel for running their instance of our proposed CPARC distributed resource allocation algorithm. Initially, every radar will transmit predetermined data on all the OFDM subcarriers except CC. After the allocation of a subcarrier to a particular (source radar, destination radar) duple is complete and this information is broadcast to their one-hop neighborhood, all neighboring radars of this radar pair will cease their transmission on this subcarrier. This technique will keep the OFDM subcarriers occupied and hence ensure a noiselike transmit waveform, even when the number of radars in the system is lower than the number of available subcarriers.

The following local and global data structures are maintained for state information: local-allocation-information (LAI) and neighbor-list $\left(N_{l}\right)$ for radar node-specific information, and global-allocation-information (GAI) for system-wide information. The LAI is used by the CPARC algorithm running in a radar node to store information on this node's pairing with its neighbors and the corresponding subcarrier(s), which were allocated to them. Whereas the GAI is indexed with all the available subcarriers in the system and every time a subcarrier is allocated, the GAI is updated and broadcast as globally available information. $N_{l}$ is used to record the nodes in the onehop vicinity of a given node. In all radar nodes, CPARC runs in an infinite loop. It performs a 4-way handshake to complete pairing, by communicating through the following seven messages. Every radar node uses the following messages:

- HELLO: to register itself with its neighbors,

- PROPOSAL: to request for pairing with a particular neighbor in response to a HELLO,

- ACCEPT: to send acceptance of pairing using a particular subcarrier in response to a PROPOSAL,

- REJECT: to send rejection for pairing using a particular subcarrier in response to a PROPOSAL,

- CONFIRM: to send an confirmation in response to a ACCEPT,

- INFO: to broadcast the GAI to its one-hop neighbors,

- OFFLINE: to tell it does not need any pairing.

Further details follow in the description of CPARC given below.

CPARC Algorithm:

Input: Number of subcarriers available per radar node, Number of overall subcarriers available in the system, Incoming message.

Output: Per-node subcarrier allocation i.e., (transmit, receive) pairing.

\subsection{CPARC: initialization}

Radar node initializes LAI, GAI, and broadcasts a HELLO message with its identity, on the $\mathrm{CC}$ indicating that it would like to also share the communication medium. It then waits for any PROPOSAL it might receive from its neighbors.

\subsection{CPARC: physical layer's channel-diversity feedback}

The analytical derivation of the probability of bit error $\left(P_{b}\right)$ versus SNR for the UWB noise-OFDM radar and its agreement with computer-based simulations has been shown in [7]. Denoting the received signal on the $l$ th subcarrier to be $Y_{l}$, the probability of bit error on $Y_{l}$ can be expressed as

$$
P_{b}\left(Y_{l}\right)=v_{l} \mathrm{Q}\left(\sqrt{\frac{\epsilon_{s, l}}{\sigma_{y, l}^{2}}}\right),
$$

where $v_{l}$ represents the magnitude scaling on the $l$ OFDM sub-carrier due to the receiving filter in the system, $Q($.) is the tail probability of the standard normal distribution, $\sigma_{y, l}^{2}$ is the variance of the additive white Gaussian noise, and $\epsilon_{s, l}$ is the signal energy of the symbol being modulated onto the $l$ th OFDM sub-carrier. From this expression, it can be seen that the reliability of transmission, even for the noise-OFDM waveform, depends on the SNR at the receiving end. Therefore, between any pair of radar nodes, choosing a subcarrier that maximizes SNR thereby minimizing $P_{b}\left(Y_{l}\right)$, is the key to exploiting channel diversity between them.

When a node running CPARC receives a HELLO, it responds with a PROPOSAL that contains a feasible set (FS) of subcarriers. FS is defined the set of all free subcarriers available for use in its GAI. Until a subcarrier is assigned to a radar pairing, it will be available in the GAI. Through this FS, it is proposing to the node that transmitted the HELLO that one subcarrier among this FS can be used for their pairing. The receiving node performs a discrete Fourier transform (DFT) on the incoming OFDM signal to decipher the data present on the CC. When it determines that it has received a PROPOSAL, it gets the channel estimate based on dummy data being transmitted on all the subcarriers of the FS present in this incoming waveform. Based on this channel estimate and the PSD on all these carriers, it will select the subcarrier that minimizes $P_{b}\left(Y_{l}\right)$, and hence will be the best to pair with its neighbor. For the AWGN or Rayleigh-affected wireless channel, between this set of (transmitting, receiving) radars, this subcarrier forms the best possible choice for pairing since its channel magnitude response is the best among all the available subcarriers. Figure 6 shows the conceptual view of this cross-layered channel information going from the PHY to CPARC in the medium access 


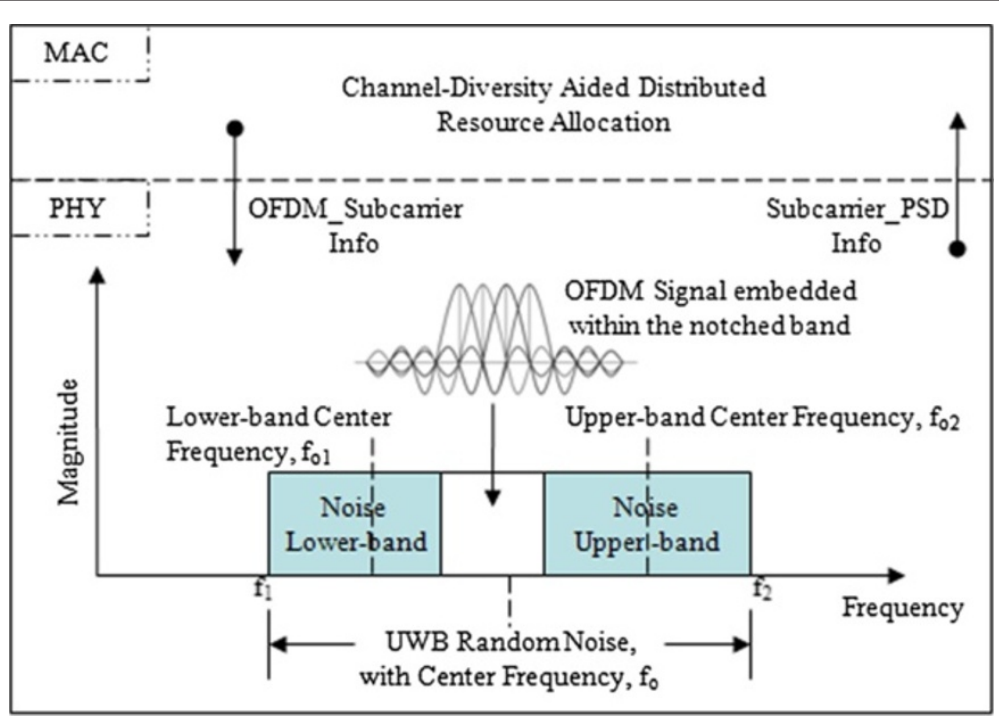

Figure 6 Depiction of the cross-layered architecture of a single radar sensor.

layer. The pairing procedure continues as described in the following section.

\subsection{CPARC: received message processing}

- HELLO: Check in the LAI if it has already paired with this new neighbor. If yes, then ignore this message. Otherwise, based on the availability of free subcarriers (by checking the GAI), choose FS and send a PROPOSAL to the source of this message. If free subcarriers are not available, then the radar cannot pair with this new neighbor.

- INFO: Copy the GAI that has come in. This information will be used in FS/subcarrier selection. When a radar gets this messages, it checks to see if its LAI matches with the information that has come in. If yes, then allocations are consistent. If not, then this radar might have missed some ACCEPT or REJECT message due to collisions, so this radar will update its LAI.

- PROPOSAL: Using the DFT performed in the receiving side and its PSD output, select the best subcarrier from the FS, and check in the LAI to see if this radar is already using this subcarrier. If yes, then further check if the corresponding source and destination pair are the same as is requested in this incoming proposal. If yes again, then this receiving radar's earlier transmission of ACCEPT might have been lost. In that case, send ACCEPT again. However, if the subcarrier is being shared with some other radar, then send REJECT. Furthermore, if this subcarrier is not being used currently, then check for availability in GAI and quota of subcarriers: if OK, send an ACCEPT message.
- ACCEPT: Check if the subcarrier for which this ACCEPT has come is already being used with some other radar, then send a REJECT message to the source. Otherwise, pair this radar with the source of the message and update the LAI, GAI. Send a CONFIRM message out to the source. If the CONFIRM is not for itself, then update the GAI about this new pairing.

- CONFIRM: Update LAI, GAI, broadcast an INFO message containing the GAI to all radars in its one-hop vicinity, to advertise this pairing. If the CONFIRM is not for itself, then use this message to update the GAI about this new pairing.

- REJECT: Temporarily unmark this subcarrier for which the REJECT came, check for other available subcarriers in GAI, and send a PROPOSAL again. If quota for subcarriers is complete, then accept the REJECT message.

- OFFLINE: Check if the source of this message is in the radar's LAI, if so, then relinquish that subcarrier and update the GAI accordingly. Send an INFO message out to distribute this information.

The output of CPARC, therefore, is an allocation that shows the different subcarriers on which every radar is paired with its neighbors. This subcarrier set forms a dedicated contention-free communication link between the radar duple. Since this algorithm runs in every radar and global allocation information is broadcast to just the one-hop neighbors, subcarrier re-use will occur across non-overlapping zones of the system, leading to efficient bandwidth utilization. The pseudo-code for CPARC is shown in Appendix. 


\subsection{Discussion}

Multi-echo issue: with the output of CPARC and Figure 5 in reference, we observe that RS2 will not be paired with another radar on the same subcarrier that RS1 uses to talk to itself. This implies $\psi_{2 e}$ will no longer be a problem when RS2 tries to decode $\psi_{1}$. Furthermore, $\psi_{1 e}$ is basically a multipath signal for $\psi_{1}$ and OFDM is inherently capable of overcoming this issue. Therefore, our OFDM-based CPARC solves the multi-echo issue.

It is also to be noted that during the initial phase of sending a PROPOSAL, there is a possibility that multiple users may send symbols on the FS subcarriers at the same time. In this situation, the $C C$ will itself result in a collision which implies the PROPOSAL will not be detected. So the receiving node will not look at the FS for the predetermined symbols at all. A node will scan the FS subcarrier's spectral density only when the message comes through to it without any collisions, which implies that no other users interfered on the $\mathrm{CC}$ or the FS subcarriers. Hence, the channel estimation performance will not be affected. Furthermore, although the $\mathrm{CC}$ is a broadcast channel which is contentionbased and prone to collisions, redundancy built-in into CPARC using $N_{l}$ and the 4-way handshake counteracts the loss of messages due to collisions and hence aids in scalability. A further improvement performed to decrease collisions on the $\mathrm{CC}$ is to allow a radar node to respond to a HELLO only if its identification number is smaller than that of the node which sent the HELLO message. This reduces the contention caused on the broadcast $\mathrm{CC}$ channel and leads to efficient packet transmissions.

\section{CPARC throughput guarantee}

Centralized algorithms with linear complexity have been designed for scheduling, channel-assignment in multi-channel, multi-hop networks [25], that attain the maximum achievable throughout region, $\Omega$. It has been shown that such solutions cannot be implemented in a distributed manner or if so, are very inefficient [13]. The problem of distributed algorithms that solve the same is NP-hard and hence relaxed throughput-optimality is aimed for. The following proposition shows that CPARC is a provably efficient distributed algorithm achieving a guaranteed fraction of $\Omega$ and tends to be marginally better than [13].

Proposition. For a network $\Pi$ and packet arrival rate vector $\vec{\lambda}$, if $\vec{\lambda} \in \Omega$ in $\Pi$, then $\vec{\lambda} / \Theta(\Pi) \in \Omega^{C P A R C}$ in $\Pi$, i.e., the throughput region guaranteed by CPARC is $1 / \Theta$ of the maximum throughput region $\Omega$ [25], where $\Theta$ is the interference degree of the network.
Proof. The noise-OFDM radar being a multi-carrier OFDM system inherently allows the radars to communicate simultaneously with more than one of its neighbors, although on separate subcarriers. Further, CPARC guarantees that a subcarrier set assigned to a radar pair will not be again used in pairing these constituent radars with their other neighboring radars. We call this a subcarrierexclusive spectrum sharing technique. Over this core subcarrier allocation strategy, we assume the use of a simple low-complexity distributed maximal scheduling flow strategy [26]. It basically ensures that if a transmitting radar $r 0$ has a packet to transmit to a receiving radar $r 1$, either $(r 0, r 1)$ or a (transmitter, receiver) pair that interferes with $(r 0, r 1)$ is scheduled for transmission; the scheduling is otherwise arbitrary. This is a valid assumption in CPARC, as the GAI developed by it in every radar has each of the neighboring radar's subcarrier assignment information that is necessary to generate the required interference relations.

An interference relationship being defined as follows: For every radar-pairing that CPARC performs using a particular subcarrier $\delta$, there is a set of radar pairings $\Gamma_{\delta}$ such that, a transmission on $\delta$ by the original radar pair and one of the pairs in $\Gamma_{\delta}$ at the same time, will not result in any useful transfer of data. The strength of CPARC is that $\Gamma_{\delta}$ will only consist of nodes which are one-hop away from either of the constituent radars of the original pairing. A non-interfering subset of $\Gamma_{\delta}$ is a subset of pairings $\bar{\Gamma}_{\delta}$ such that any two pairings in this subset do not interfere with each other. The interference degree $\Theta(\delta)$ [26] of pairing $\delta$ is, therefore, defined as (i) the maximum size of the subset $\bar{\Gamma}_{\delta}$ if $\Gamma_{\delta}$ is non-empty and (ii) 1 if $\Gamma_{\delta}$ is empty. The interference degree of the whole network $\Theta$ is the maximum interference degree over all the pairings. The interference degree of the system is directly dependent on the physical interference model [13] and thus is independent of the exact network topology. Further, it is well known that the efficiency ratio of an algorithm is the largest number $\eta$ such that this algorithm can stabilize the system under any load $\vec{\lambda} \in \eta \Omega$. Given the above, and the pair-wise interference relations developed from GAI, it follows directly from Theorem 1 in [26] that for our system $\Pi$, if $\vec{\lambda} \in \Omega$ in $\Pi$, then $\vec{\lambda} / \Theta(\Pi) \in \Omega^{\text {CPARC }}$ in $\Pi$, i.e., CPARC can guarantee an efficiency ratio of $1 / \Theta$, which is comparable to [13].

Furthermore, intuitively, the upper bound of $\Theta$ as mentioned in [26] will not be tight in our system, due to the OFDM-based multi-carrier advantage. Using the subcarrier-exclusive spectrum sharing technique, the common end point interference constraint no longer prevails. Due to this, $\Gamma_{\delta}$ decreases, leading to a decrease in the upper bound of $\Theta$, and hence an increase in the efficiency ratio of our algorithm. This makes CPARC marginally better than [13]. 
8 Simulation results and performance evaluation To validate the concepts of the UWB noise-OFDM radar system presented above, simulations were performed for communications and radar performance using MATLAB ${ }^{\circledR}$, while CPARC was simulated using the OMNET++ simulation framework [27]. The UWB noise signal covered the $1-2-\mathrm{GHz}$ band, with the notch band over 1.2-1.3-GHz. A 253-tap finite-impulse-response (FIR) equi-ripple BPF with a transition region bandwidth of approximately $50 \mathrm{MHz}$ and a pass-band magnitude ripple of $\pm 0.5 \mathrm{~dB}$ was used in the receiver. The input parameters to these simulations are the number of subcarriers being used (which is 32 to cover a $100-\mathrm{MHz}$ notch), the number of symbols per carrier (which is 50 in our case), the bin length (1024), sample frequency of $5 \mathrm{GHz}$, and the channel SNR. Based on this required channel SNR, appropriate noise power was calculated and added to the transmitted signal.

\subsection{Multi-user radar-communications performance}

To study the multi-radar data communication characteristics of the noise-OFDM netted radar, we simulated this system as described in Section 2 to plot the BER versus SNR curves. We considered a multi-radar system, with varying degrees (number of one-hop neighbors). Suppose a radar $i$ has neighbors $j$ and $k$. We assume that $i$ is allowed to communicate with $j$ on the odd-numbered subcarriers, and with $k$ on the even-numbered subcarriers. Both $j$ and $k$ individually produce their noise-OFDM waveforms and transmit them to $i$. Upon receiving their transmissions as a composite AWGN-affected waveform, $i$ demodulates the incoming signal and separates $j$ 's and $k$ 's data bits. Figure 7 shows the BER versus SNR performance. Comparing noise-OFDM to a pure OFDM signal, we observe that due to the high error floor (noise-OFDM, Degree 2 in Figure 7), degree of a radar node does significantly affect the noise-OFDM system. However, utilizing a 3carrier data redundancy (DR) [7] technique (noise-OFDM w/DR, Degree 1 and 2 in Figure 7), we observe that this floor can completely be removed and the performance improves remarkably, and a BER better than $1 \times 10^{-5}$ for an SNR of $12 \mathrm{~dB}$ is achieved. This result is from our experimental simulations with multiple users in the system and DR being used. At this same BER, there is still a $10-\mathrm{dB}$ degradation in the worst case, compared to pure OFDM's performance. This is the price paid to achieve the PHY security that this system presents to the transmit waveform.

\subsubsection{Notch and BPF design}

The significant degradation of BER performance seen in Figure 7 is also due to the filtering effect of the side lobes of the OFDM signals. To overcome the BER degradation due to the filtering affect, good design of the notch and BPFs in the transmitter and receiver subsystems of the radar is required. The UWB noise-OFDM Radar's transmit signal is a composite signal consisting of a "UWB noise" waveform surrounding the "OFDM" waveform. As for the notch filter in the transmitter, it is to be designed to not let spectral gaps be visible between the UWB noise signal and the OFDM signal, and in the other extreme, to not overlap the OFDM signal. In our design, the UWB noise

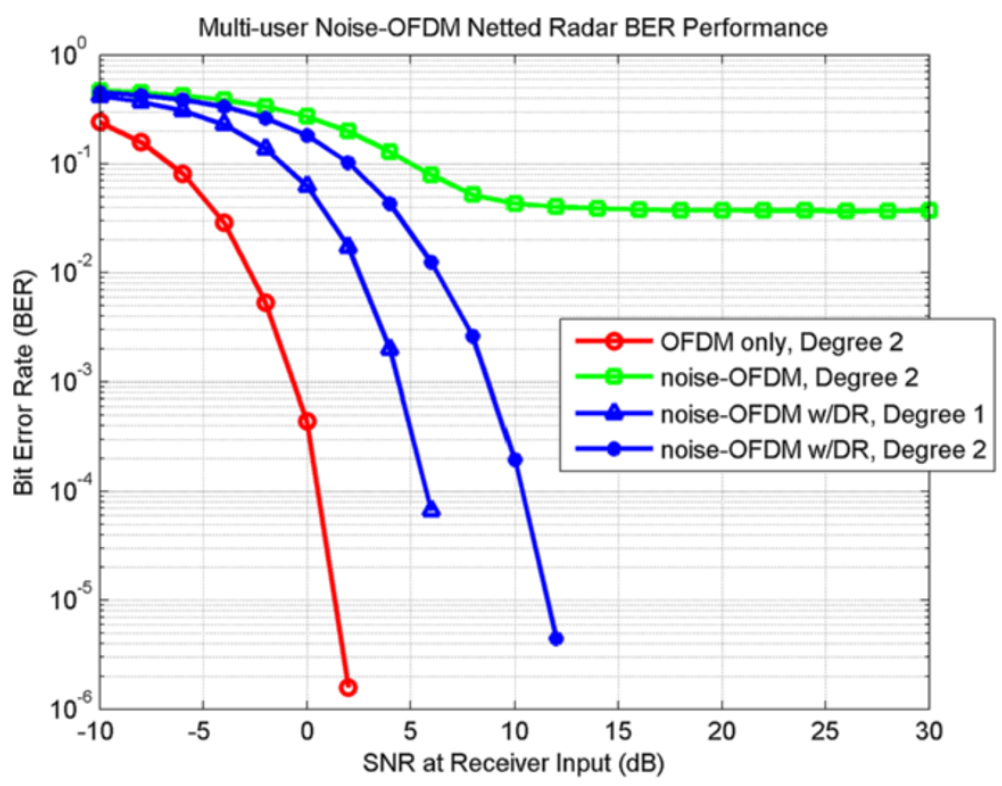

Figure 7 Noise-OFDM multi-radar BER versus SNR in an AWGN channel. 
signal is notched first and then an appropriate number of sub-carriers for the OFDM signal are chosen, so as to obey both the conditions above. Having done that, the BPF in the receiver subsystem becomes a more crucial component deciding the performance of this radar system. Any mismatch between the bandwidth of the BPF (BPF seen in Figure 4) and the embedded OFDM signal's bandwidth can lead to two cases of BER concerns:

1. If the bandwidth of the BPF is greater than the OFDM signal's bandwidth, then portions of the UWB noise waveform surrounding the OFDM signal is drawn into the OFDM demodulation process, thus causing unwanted additive UWB noise signal energy to be present in the demodulator output.

2. If the bandwidth of the BPF is less than the OFDM signal's bandwidth, then the resulting truncation of the OFDM signal reduces the orthogonality of the OFDM subcarriers, causing ICI.

Therefore, if the BPF is designed appropriately such that the BPF's bandwidth is larger than the OFDM signal's bandwidth, then Case 1 mentioned above results. Due to the additive nature of the UWB noise, this does not affect the orthogonality of the subcarriers, hence causing no ICI in the system. Since the subcarriers on which data will be ported are known, the OFDM signal's spectral bandwidth is also known. Therefore, a BPF that satisfies Case 1 mentioned above is appropriately designed for use in the receiver subsystem, ensuring no ICI is caused. Our earlier study [7] details these physical layers aspects of the noiseOFDM waveform with respect to spectral equalization, noise-like properties, reliability, and covertness.

In [7], we have also derived the BER for this radarcommunications system theoretically. Our BER analysis is customized to the noise-embedded OFDM signal. This is a unique method, since the non-ideal BPF in our receiver, based on its pass-band design, either truncates the OFDM signal or allows out-of-band noise signal causing errorfloor and magnitude ripple related issues in the demodulation process. And these effects are well modeled in the analysis. A close match between theory and simulations has also been shown. Based on the same validation of the simulation setup, we derive these further results for multiuser radar communications systems, which conclude to have a BER of $1 \times 10^{-5}$ for SNR of $12 \mathrm{~dB}$. Theoretical enhancement of the above derivation to a multi-user scenario is a future scope for this study.

\subsection{Radar operations: range and doppler resolution}

Target ranging, Doppler estimation, range resolution, and Doppler resolution are the important metrics for measuring the performance of a UWB noise radar. It was already shown in [7] that the range estimation performance of the UWB noise-OFDM radar is unaffected by the presence of an OFDM signal in the transmit waveform. In this article, for further verifying radar performance in the presence of embedded communications, we simulated the $\mathrm{AF}$ expressed in Equation (5). From the AF plot, the range and Doppler resolution of the noise-OFDM radar can be inferred. Range resolution $\Delta R=c / 2 \beta$, where $c$ is the speed of light $\left(3 \times 10^{8} \mathrm{~m} / \mathrm{s}\right)$ and $\beta$ is the bandwidth. $\beta$ also corresponds to the inverse of the first zero-crossing of the AF curve along $\tau$ axis. The system auto-correlation functions are as given in Equations (19) and (20). We used an integration time of $50 \mathrm{~ms}$, a notch bandwidth of $300 \mathrm{MHz}$, and a center frequency of $1.45 \mathrm{GHz}$. The squared outcome (reduces side-lobes) of the convolution in Equation (5) at time $t=T$ was stored for each $\alpha$ and $\tau$. The resulting $\mathrm{AF}$ and its slice along $\tau$ axis is plotted in Figures $8 \mathrm{~b}$ and 9, respectively. From Figure $8 \mathrm{~b}$, it can be noted that the main lobe width (proportional to range resolution) remains relatively unaffected compared to a conventional UWB noise radar's AF main lobe in Figure 8a [23], although its side lobes are slightly higher. Figure 9 in further detail compares the range resolution of a UWB noise radar with a UWB noise-OFDM radar of varying notch bandwidths. Since our goal is to have concealed coexistence of data with radar, we observe that a reasonable trade off can be reached between efficient wireless communications and covert radar operations: e.g., as seen in Figure 9, a $300-\mathrm{MHz}(30 \%)$ communications bandwidth is available with $45-\mathrm{cm}$ radar range resolution in the UWB noiseOFDM radar compared to $15-\mathrm{cm}$ range resolution of conventional UWB noise radar with no communications ability. It is also seen that the range resolution improves as the notch bandwidth (embedded OFDM bandwidth) increases, i.e., $15 \mathrm{~cm}$ for $900-\mathrm{MHz}$ notch. However, as the embedded OFDM bandwidth increases, the overall noiselike behavior of the signal decreases [7], hence reducing its covertness.

Our earlier studies have shown that spectral fragmentation of the radar frequency band causes no essential distortion to the main-lobe of the point spread function if the fragmented gap portion is not too excessive, i.e., $\leq 30 \%$ [28]. It shows that by spectral fragmentation, range resolution is unaffected but the problem is that the range side-lobes increase from -36 to $-20 \mathrm{~dB}$ for fragmentation greater than $30 \%$, thereby causing ghosts and artifacts in the image generated of the target. Hence, the spectral gap should be less than $30 \%$ to not adversely affect radar performance. The novelty of our approach has been to embed data into this fragmented radar spectrum. The AF derivation presented herein, therefore, measures and quantifies this enhancement. As seen from the AF plots, embedding OFDM data into the radar spectrum by spectrally carving out up to $30 \%$ of the UWB bandwidth does lessen the radar range resolution. However, the velocity resolution 


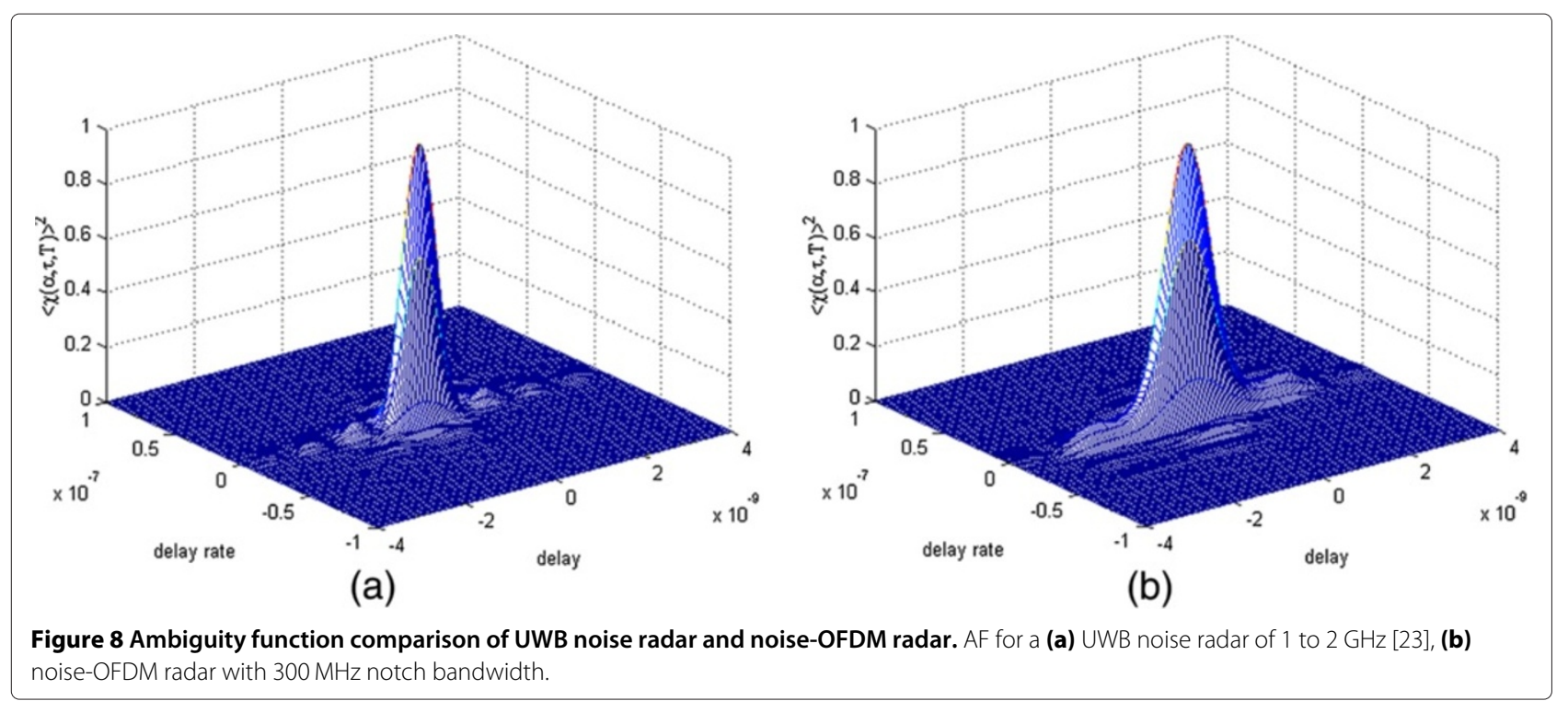

(Doppler, a slice of Figure $8 \mathrm{~b}$ along the $\alpha$-axis) remains unaffected by the varying sizes of the notch bandwidth or the data embedded into it, as shown in Figure 10. This is expected since the velocity resolution depends only on the integration time [23] and not on the waveform bandwidth. As range and Doppler estimation are the primary objectives of a radar, we can now observe that the radar performance is affected, albeit slightly. The tradeoff therefore, is to relax the range resolution requirement, and use it add wireless secured multi-radar networking into the system. Furthermore, this range resolution lost can be gained back by increasing the bandwidth of the overall transmitted signal.

\subsection{CPARC performance validation}

CPARC was implemented and tested on a multi-hop radar network with varying number of radar nodes and node degrees (number of neighbors). On the OMNET++ framework a broadcast wireless channel was designed where the UWB noise-OFDM radars as wireless nodes could send/receive packets. This channel emulates the CC. Every node has a packet transmit/receive module to

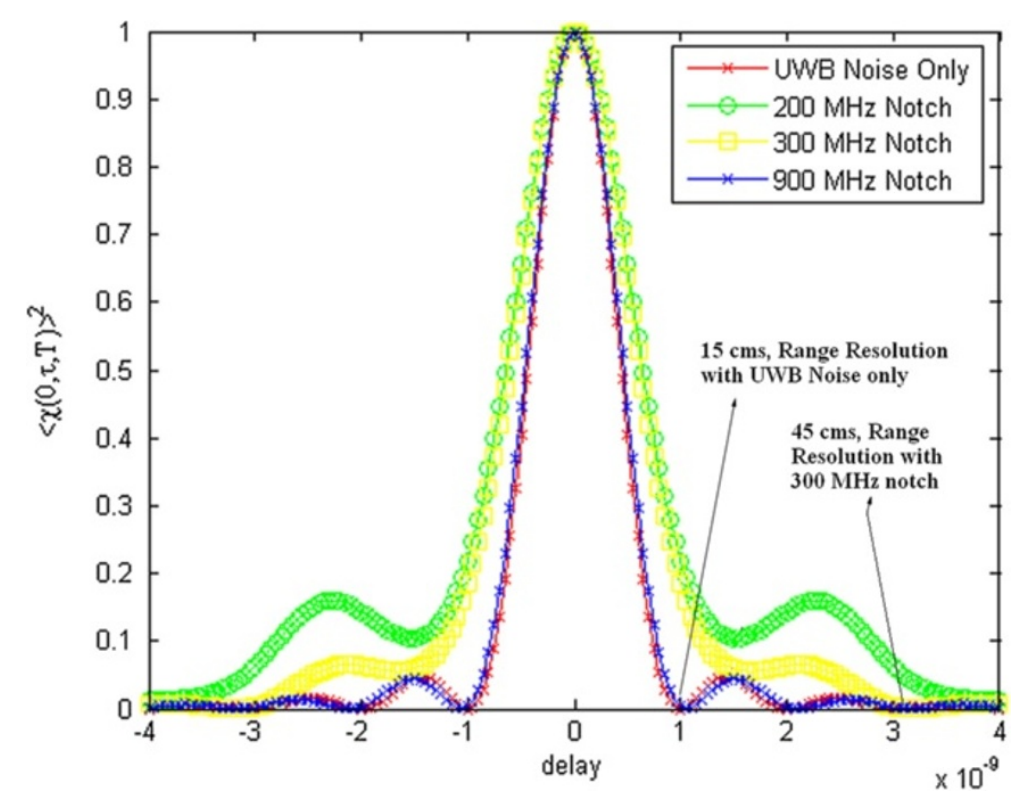

Figure 9 Ambiguity function showing range resolution. For $\alpha=0$, the 2-D AF plot showing range resolution. 


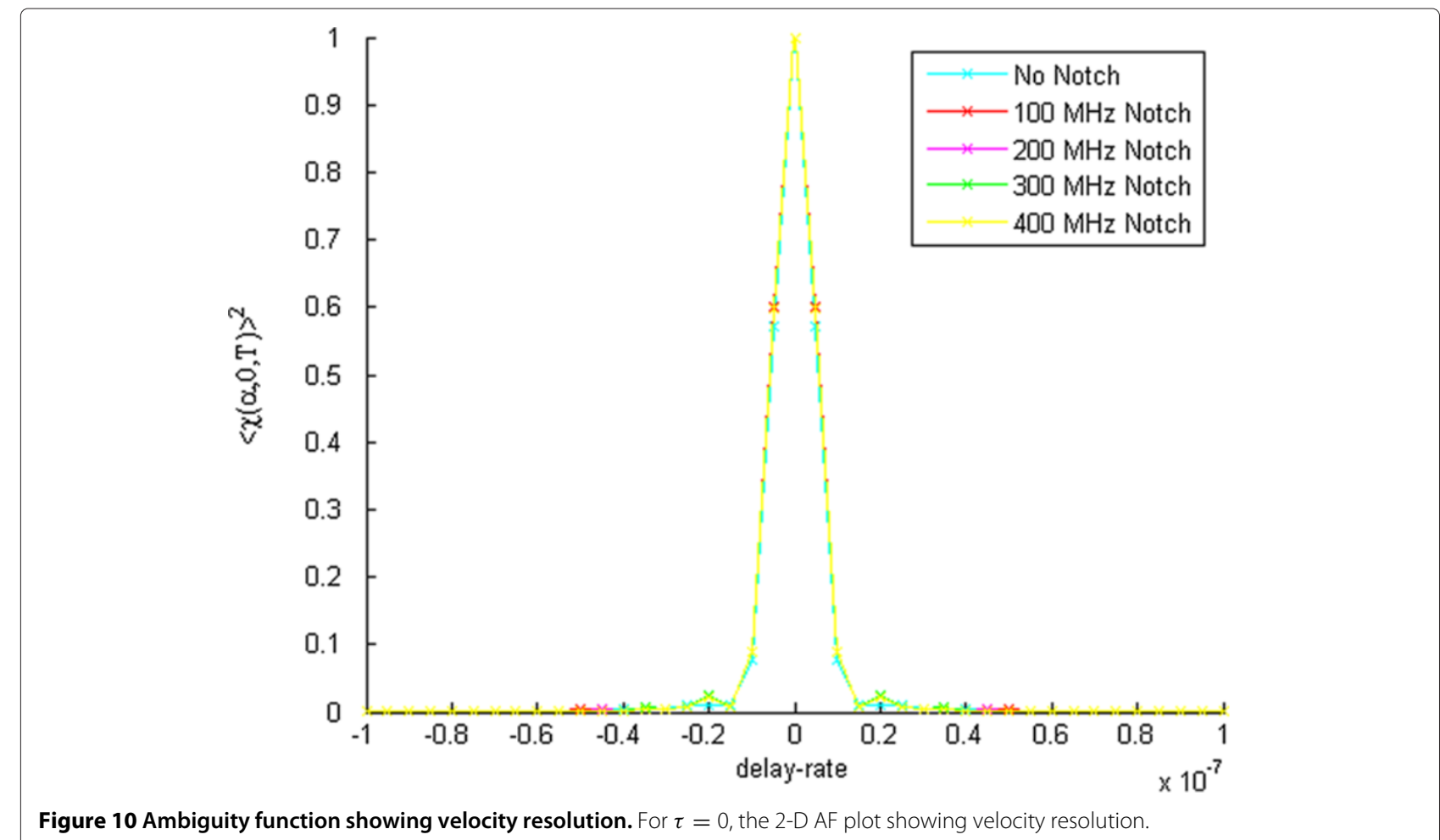

transmit/receive packets to/from the wireless medium. Packet transmission rate $(9.6 \mathrm{kbps})$, propagation delay (10 ms), packet length (952 bits), slot time (100 ms), packet transmission duration $(100 \mathrm{~ms})$, collisions due to simultaneous transmission in the wireless medium, multi-hop topology of the radar network, etc., were appropriately modeled based on the verified Slotted ALOHA broadcast network in OMNET++. The simulation architecture of CPARC is shown in Figure 11. Here, message packets from the receive module were directed to a FIFO receive queue, from where they were grabbed by CPARC for processing. Outgoing messages were stored in the transmit queue for the transmit module to flush out the packets into the wireless medium. The wireless broadcast network has parameters as mentioned above. Within OMNET++, during any packet transmission in the node, if one or more packets arrive to the same node, it is treated as a collision for both the transmitter and the receivers. Similarly, while

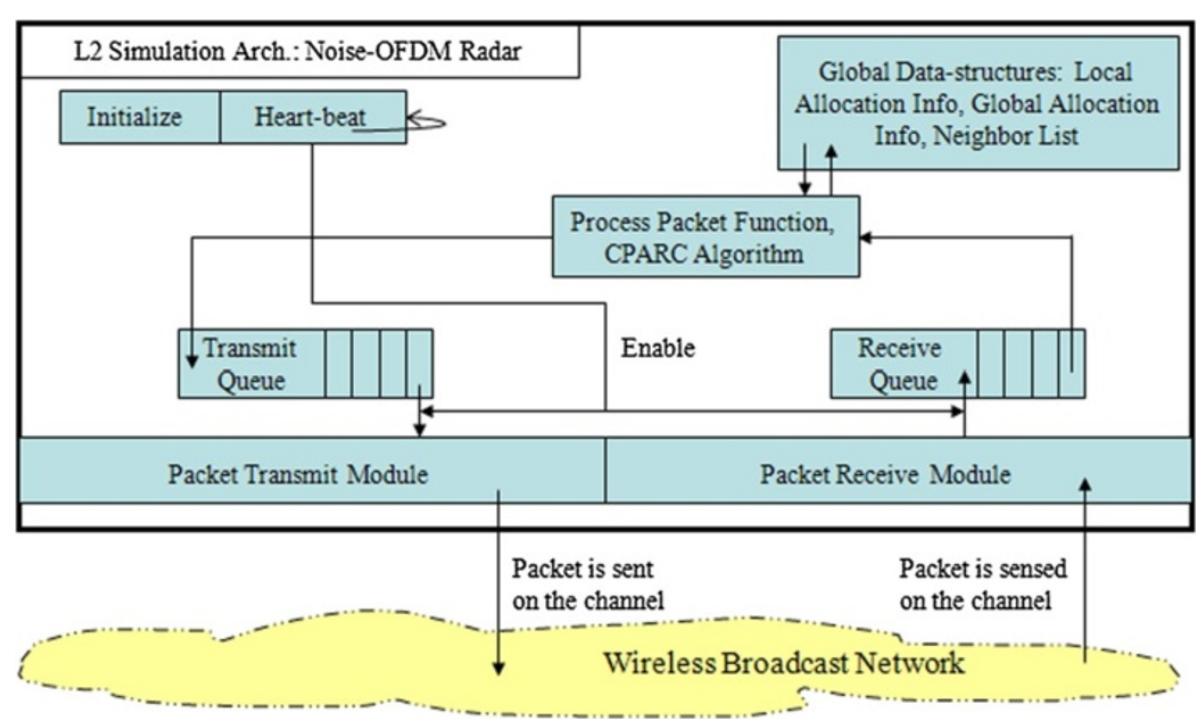

Figure 11 Simulation architecture for CPARC in the UWB noise-OFDM radar. 


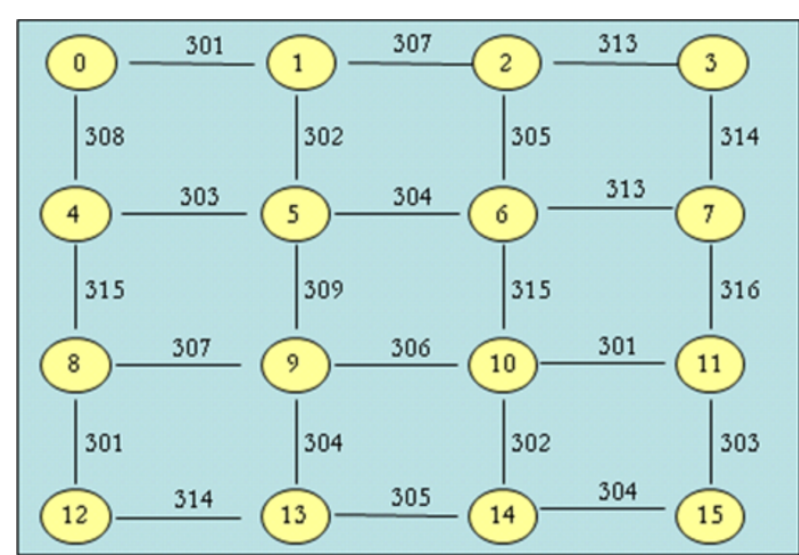

(a)

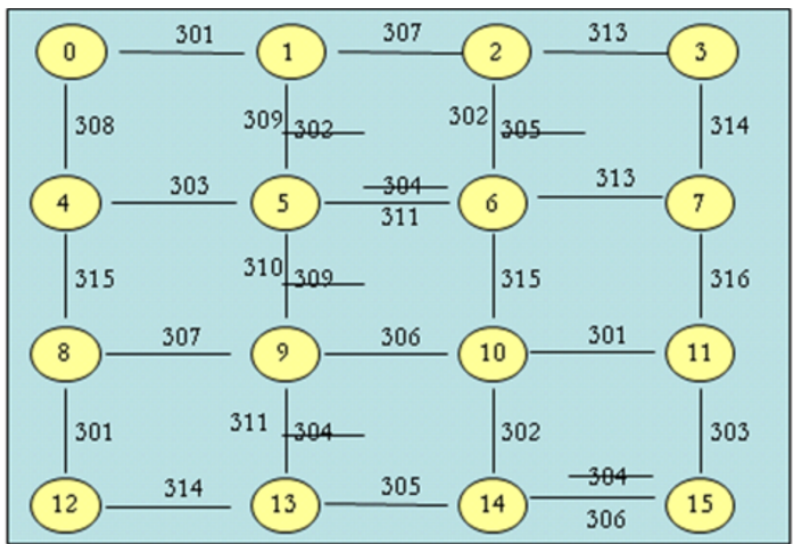

(b)

Figure 12 CPARC output for a regular grid topology. CPARC output: (a) Subcarrier allocation for a 16-radar network with node degree's 2, 3 and 4. Example $(R O, R 1)_{301}, R 0$ and R1 paired on subcarrier 301 . (b) With subcarrier 304 becoming unavailable, CPARC dynamic re-allocation output.

receiving a packet, if one or more packets arrive, then it is treated as collision for the transmitters and the receiver. The overall number of subcarriers available in the whole network was chosen to be 18 and the number of subcarriers available per node was 8 , based on the OFDM bandwidth and DR requirements.

\subsubsection{CPARC distributed channel-aware allocation}

Initially, we tested CPARC on a 16-node square topology as in [13] and later, on random topologies with varying number of radar nodes (up to 90 nodes) and node degrees (2 and 4). It was observed through our simulations, which were averaged over 20 independent runs, that in each test case: (i) the distributed algorithm at every radar generated consistent output, i.e., if radar $i$ showed that it was paired with radar $j$ on subcarrier $K$, the same was observed in the output of radar $j$ with respect to $i$, and (ii) all the radars in the network were paired with as many of their radio-range neighbors as possible, subject to the availability of free subcarriers and no contention. The result of the regular square network topology is shown in Figure 12, while an irregular 20-radar random topology is shown in Figure 13. We named the available sets of subcarriers sequentially from 301 to 318 , and the radar nodes from R0

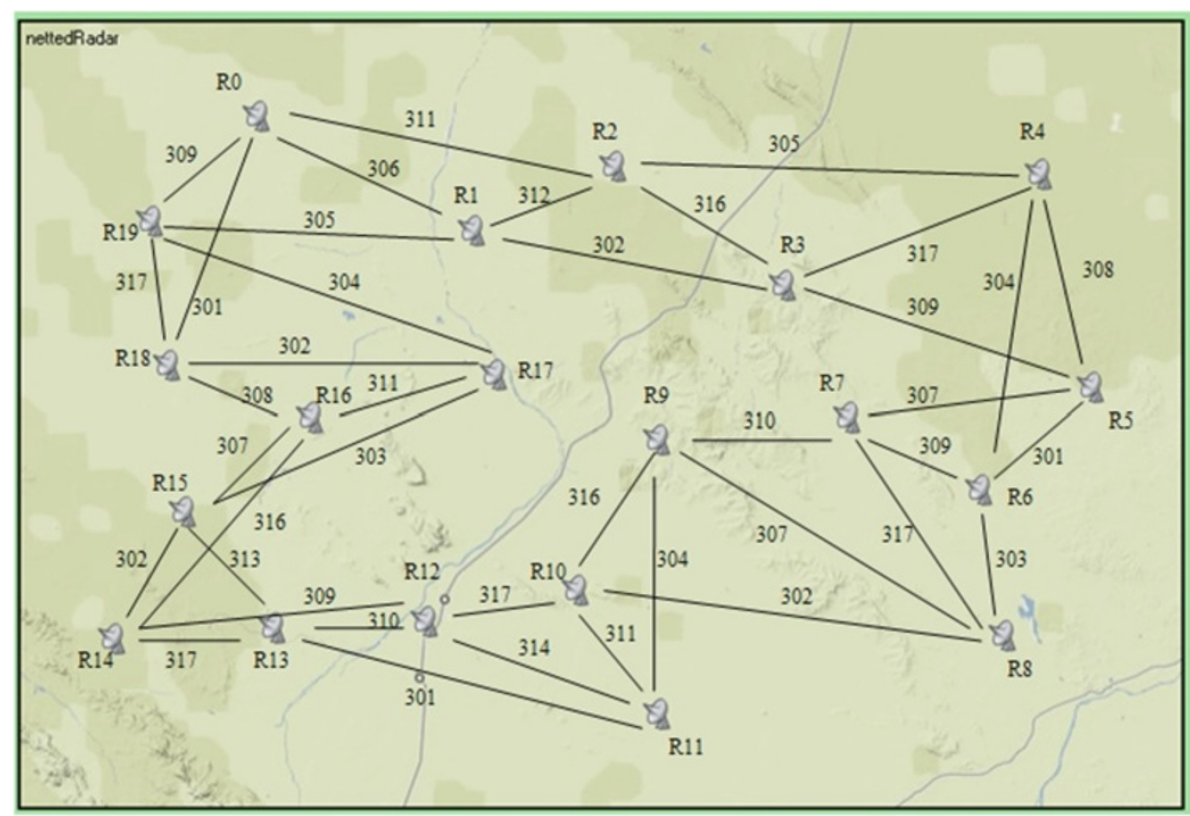

Figure 13 CPARC allocation output for a random wireless network. 
through to R15. The output shown in Figure 12a, states that $\mathrm{R} 0$ and $\mathrm{R} 1$ are paired to communicate on 301, R0 and R4 on 308 etc. Although most of the radar pairs get contention-free allocations, we also see here that subcarrier 304 being used by radar pairs (R5, R6), (R9, R13), and (R14, R15) could possibly interfere with each other. However, as stated earlier, whenever a radar gets a broadcast from its neighbor that it is using a particular subcarrier, then it ceases its own transmission on that subcarrier. So in this case, when R9 gets the broadcast from R5 asserting its use of 304, then R9 ceases its transmission on 304 or vice versa. Furthermore, this does not disconnect R9/R5 from the network, because if R5/R9 is hearing of this contention, it implies that R9/R5 is already connected to R5/R9. Same is the case with subcarrier 313. Therefore, as observed from these results, CPARC is seen to satisfy all constraints of subcarrier allocation, allowing all radar pairs to be assigned contention-free communication links and thus verifying its correctness.

\subsubsection{CPARC control channel performance}

As observed from the above CPARC algorithm, the radar nodes do contend to access the $\mathrm{CC}$, also called the control channel. But once channel allocations are complete, then contention-free sub-carriers are established between every radar and all of its one-hop neighbors. This essentially creates a contention-free communication channel between the radars. As the wireless channel varies and radar's get disconnected on a particular sub-carrier(s) that they are paired on, then CPARC automatically again uses the contention-based control channel to get the radars paired on some other available and good channel. Therefore, for data traffic, it is a contention-free system. However, for control traffic, it is still contention-prone. Slotted Aloha is a MAC mechanism where there is no carrier sense happening. This is a prime requirement for the Noise-OFDM system, as all the sub-carriers in the OFDM signal will be either carrying real data or fake data or UWB noise. Therefore, trying to sense the channel to decide on transmission is not possible and hence slotted Aloha is best suited in these conditions. However, slotted Aloha in itself is not very efficient, with only $36 \%$ efficiency of channel utilization. If all the radar nodes use slotted Aloha, then the utilization of the channel cannot be more than $36 \%$. Our approach presents a solution where the channel utilization will be $100 \%$, i.e., dedicated contentionfree channel assignments are accomplished across the radar network. CPARC enhances slotted Aloha by utilizing the multiple sub-carrier advantage that OFDM provides. In this multi-carrier enhancement over slotted Aloha, we modified it to randomize the latency in selecting the next slot for transmission, and use it as a control channel protocol to build a channel-diversity-aware distributed channel-assignment algorithm that is designed to develop a contention-free multi-hop network of radars. We assume here that the different radar nodes are time synchronized, i.e., they all have aligned clocks. To the best of the authors' knowledge, no other MAC protocol can be used in the noise-OFDM system due to the reasons detailed in Section 2. This section covers the performance analysis of CPARC in the light of success rate and latency for accessing the control channel. We define success rate as the average overall packet delivery ratio in the entire network. Figure 14 shows the packet delivery ratio versus the number of nodes in the network.

Here, we ran CPARC on an increasing network scale, nodes in the network increasing from 10 to 90 . For every set of nodes, after CPARC produced the final set of subcarriers allocations for all the radar node pairs in the network, we collected statistics on the total number of packets that were generated, the total number of packets that collided and the total number of packets that were correctly received, overall in the entire network. The ratio of the correctly received packets to the number of packets transmitted gives us the packet delivery ratio and hence the success rate of the complete network. For every set of nodes, the results were averaged over 20 runs. An essential point to note is that instead of sending the packet at the next slot interval, we randomized the latency and based on a uniform probability distribution, every radar node will randomly delay its transmission (instead of using the next available slot for transmission, a random delay is observed and then the next available slot is used), so collisions can be minimized. The results are shown in Figure 14. When this latency per node was based off a uniform distribution from $(0,2)$, we observed good packet delivery ratios, as seen by the blue and red bars in the figure. However, when this random latency was reduced, to $(0,1.75)$, it can be observed that the packet delivery ratio reduced, as seen by the green and purple bars in the plot. This was quite drastic for node degree 4. From this figure, we can observe that success rate in this modified slotted Aloha-based control channel is indirectly proportional to the latency that the nodes are allowed to carry, i.e., by introducing a random delay in selection of the next slot for transmission, packet delivery ratio is considerably high. As was mentioned in Section 2, contending for the channel or trying to sense the channel before transmission is not possible in the noise-OFDM waveform case. Therefore, this enhanced slotted Aloha technique is chosen as a base layer for the CC, over which CPARC is implemented. Of course, sending the message directly is basically setting this randomized delay to 0 . But as seen from the plot above and our further simulations, reducing this delay does drastically decrease the success rate of the system and hence is not viable.

Another important inference that can be drawn from Figure 14 is that the success rate is almost constant 


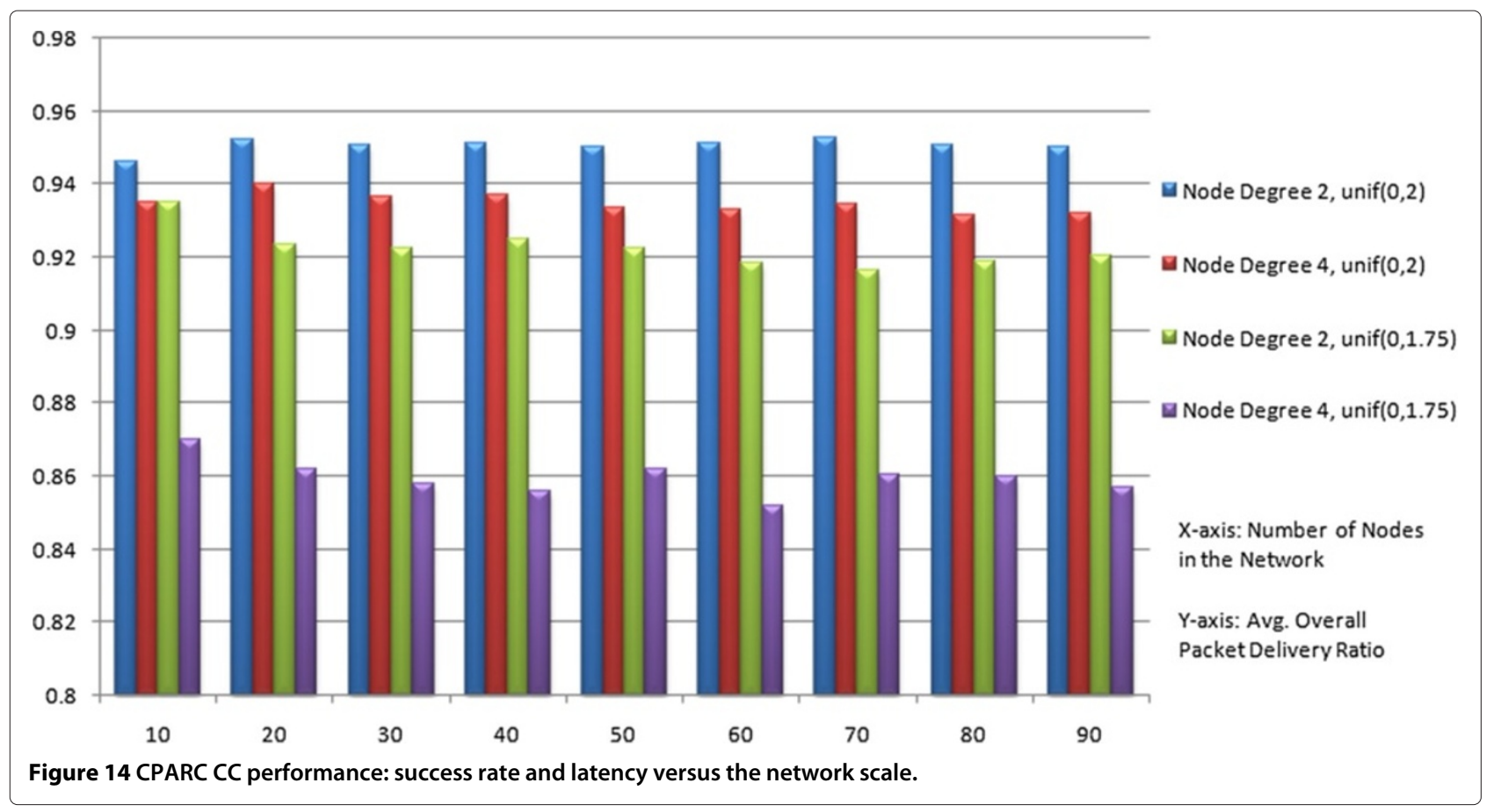

with increasing number of nodes in the network, for any latency or node degree. Since the success rate is calculated after successful completion of sub-carrier allocations across the entire network, this further goes to prove the distributed nature of CPARC and its ability to work on a multi-hop-distributed scenario.

\subsubsection{CPARC dynamic subcarrier re-allocation}

In order to test performance in lieu of wireless channel failures, we introduced faults simulating channel (subcarrier) failures in our system. Using the above-mentioned 16-node square topology, after the initial allocation is complete, we simulated an event that subcarrier set 304 becomes unavailable. The resultant dynamic update produced by CPARC is shown in Figure 12b, where we now see that no radar pairs use 304. This holds true for multiple subcarriers and link failures too. Here, a subcarrier failure is the event that a particular subcarrier is unavailable to the whole system, whereas a link failure is the event that the specific pairing between any two given radar nodes fails due its associated subcarrier being unavailable locally.

We present Figure 15 to verify scalability and runtime performance of dynamic re-allocation in CPARC. The ordinate in is the ratio of the number of messages needed to perform re-allocation to the number of messages needed for the initial assignment. First, it can be seen that CPARC overcomes both link and subcarrier failures. Second, even for 50\% link failures (12 links failing), CPARC only needs $24 \%$ of the messages to complete reallocation as compared to the initial assignment. The maximum number of subcarrier failures that CPARC can tolerate is directly proportional to the total available subcarriers in the system. Furthermore, when any radar goes offline or new radars come into the network, our simulations showed that CPARC dynamically reallocates subcarriers, respectively, subject to the availability of free subcarriers. These results confirm CPARC's ability to dynamically overcome channel and radar node failures, allowing the system to adapt efficiently to changing topology and wireless channels.

\subsection{Performance comparison of CPARC with centralized edge-coloring algorithm}

Scalability of resource allocation solutions is an important concern, especially in an ad hoc network setup, where the number of nodes deployed could vary hugely. In order to test the scalability of CPARC, we compared the runtime performance of CPARC (time taken to complete allocation) with a similar but centralized algorithm-the edge-coloring graph algorithm [29]. The edge coloring of a graph is the assignment of colors to the different edges such that no two adjacent edges are given the same color. This is similar to the subcarrier allocation problem, wherein no adjacent receiver-transmitter pairs should be allocated the same subcarrier. Furthermore, we also note that the edge coloring problem is constrained lesser than the problem that CPARC deals with since the former does not have to take care of the hidden terminal problem. Since CPARC was implemented on an event-driven OMNET framework, the centralized edge-coloring algorithm was also implemented on the same framework. 


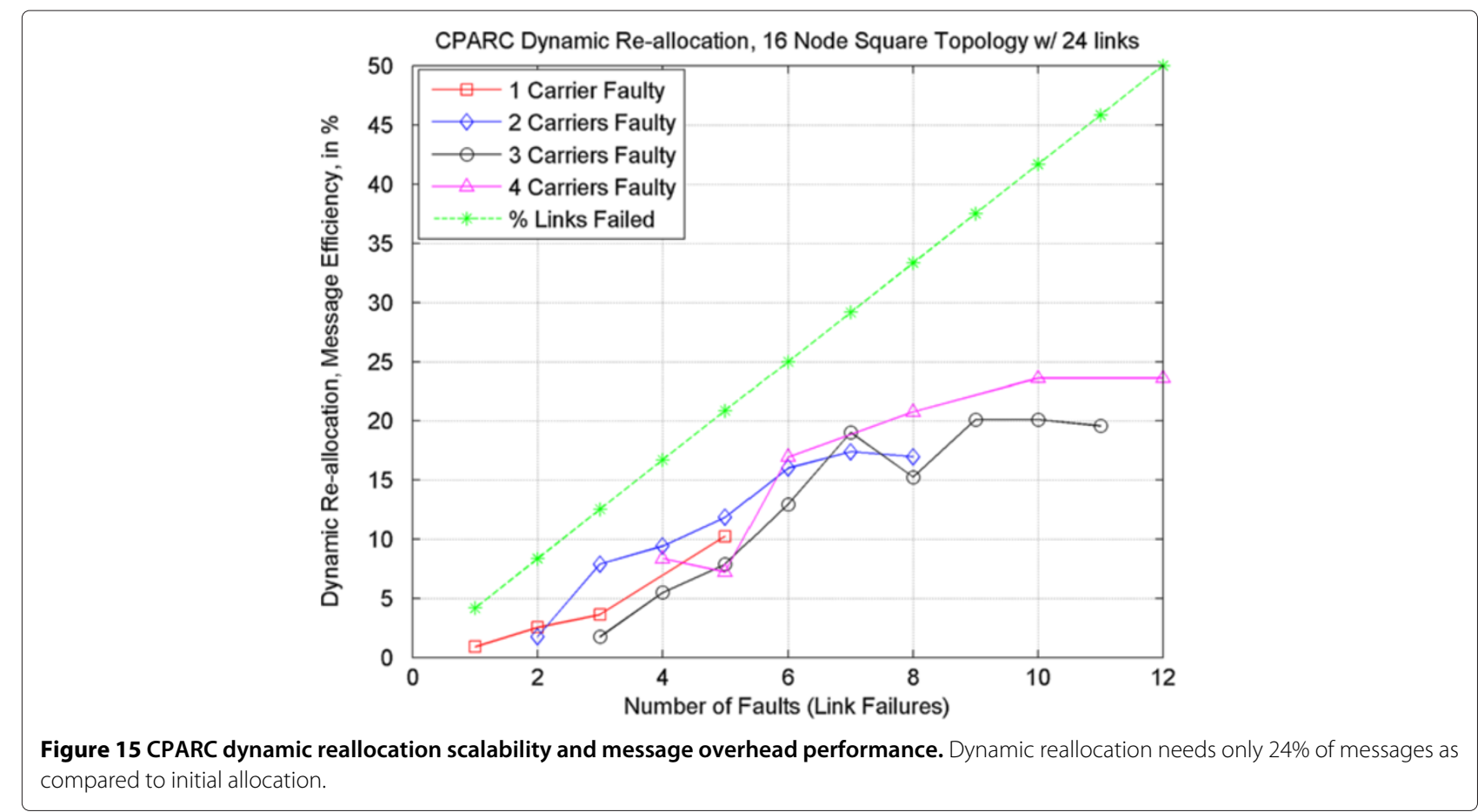

However, its running time was not measurable because the centralized algorithm did not produce any packettransmissions or network-based events which were being used to record its running time. Therefore, we ran an efficient implementation of the edge-coloring algorithm separately on a simple UNIX machine and compared our distributed solution with it based on asymptotic order of growth analysis.

Our simulation results are shown in Figures 16 and 17, where Figure 16 relates to the distributed CPARC algorithm and Figure 17 relates to the centralized edgecoloring algorithm. As can be seen from these plots,

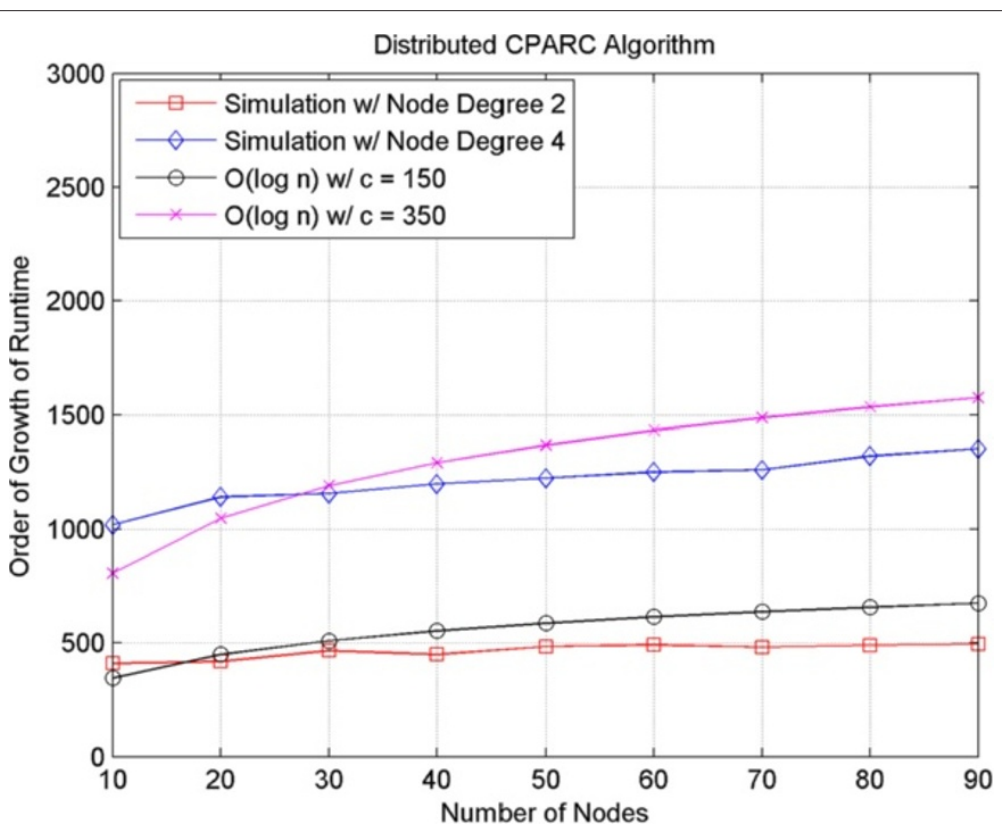

Figure 16 Scalability test for Distributed CPARC. Scalability test for CPARC to compare with centralized edge-coloring algorithm. 


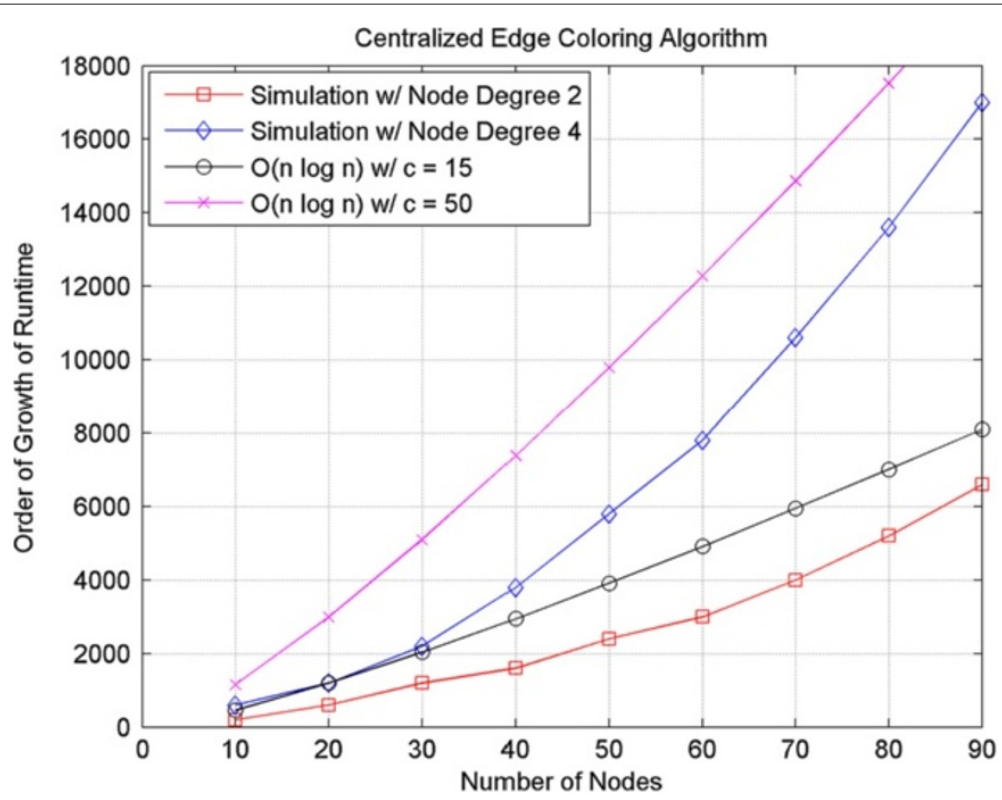

Figure 17 Scalability test for centralized edge-coloring algorithm. Scalability test for centralized edge-coloring algorithm to compare with CPARC.

CPARC's runtime order of growth is logarithmic in the number of nodes, whereas the centralized algorithm has an $O(n \log n)$ growth, where $n$ is the number of nodes. This behavior can be explained as follows. The distributed algorithm has a lower order of growth since the number of nodes a particular node has to communicate with to setup its subcarrier assignments is largely dependent only on its node degree (as can be seen in Figure 16). Hence, even though the number of nodes in the entire network might increase, the subcarrier allocation is subject only to the number of nodes in the neighborhood of a particular node. In contrast, it can be seen in the centralized algorithm that there is just one node running the whole algorithm. Hence, it can be concluded that our distributed solution is appropriate for an ad hoc network of radar nodes.

\subsection{Improved performance of CPARC over distributed frequency-hopping OFDM}

Here, we compare the performance of our distributed CPARC algorithm with another existing method in the literature, Frequency-hopping (FH) OFDM [30]. FH-OFDM is a recent and promising technique used to achieve frequency diversity gain in OFDM subcarrier allocations. In this method, every radar can choose a random seed and inform its one-hop neighbors of this seed. Then, based on this seed, it will randomly generate a sequence of subcarriers and transmit according to that sequence on consecutive time slots (assuming time is slotted). Since other radars in the vicinity know the seed of this pseudorandom sequence, they can follow along and receive the transmissions. This can happen in all the radars of the system. This method leads to good frequency diversity. This is because the radars are frequency hopping frequently, so they will not remain in any faded frequency for a long time.

However, for maintaining covertness, the radars in our system need to transmit on all the subcarriers, otherwise, the notch in the spectrum will easily be visible. So, a radar needs to be transmitting some random data on all the other 31 subcarriers, to maintain covertness. However, if it does do so, then it can bring about collisions on frequencies which are being used by neighboring radars. Although, initially, all the radars exchange their seed information with their neighbors, every radar will now need to maintain three things on a slot-by-slot basis (i) the frequency that it needs to transmit on, (ii) the frequencies of its neighbors that it should not transmit on, and (iii) the frequencies of its neighbor's neighbors (hidden terminal problem) it should also not transmit on. This third constraint above is motivated by the fact that if the 2nd hop neighbors change, then that information will not easily be known to the original radar, thereby, leading to potential collisions on the subcarriers.

We can now observe CPARC's allocations. Again, for maintaining covertness, a radar will not transmit on subcarriers that are being used by its neighbors. It will, however, transmit on all other subcarriers. For any unauthorized sensor in the vicinity, the complete spectrum will still appear noise-like. In the event that the 2nd hop neighbor moves away, a disconnect happens between the radars and all the neighbors are explicitly informed of this 


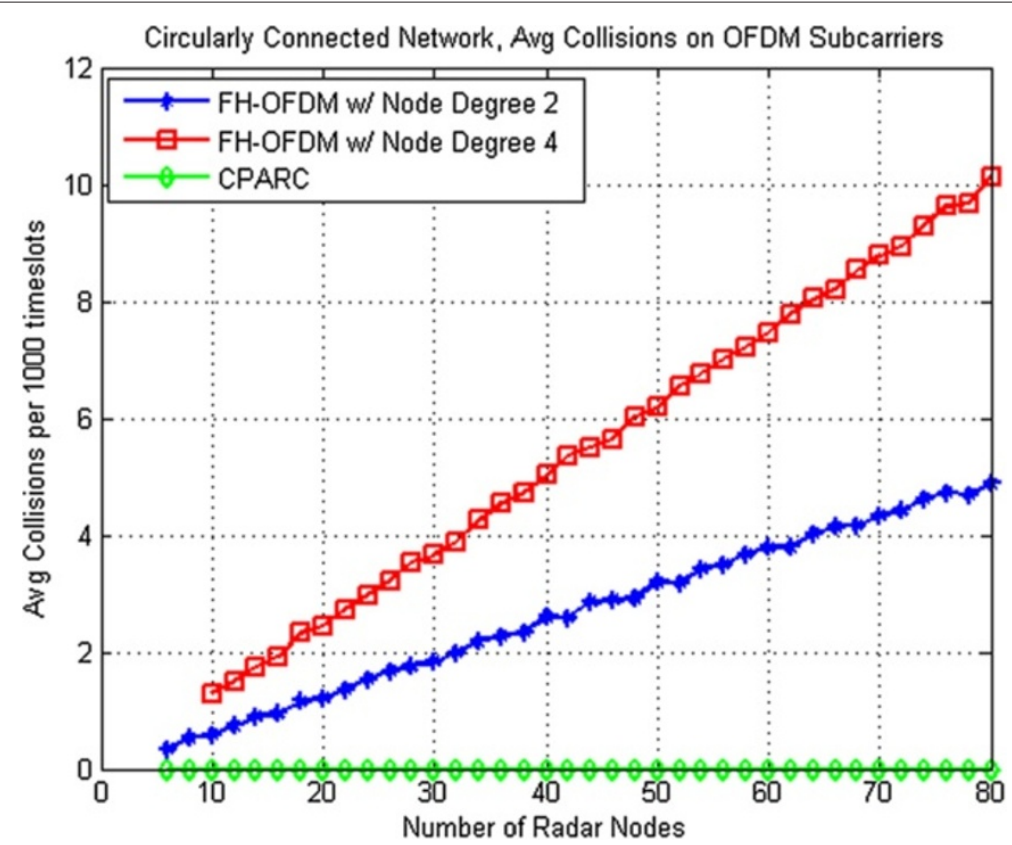

Figure 18 Average collisions comparison: CPARC against distributed FH-OFDM.

through a broadcast. Therefore, collisions are automatically being avoided in CPARC's case. Figure 18 depicts this very aspect quantitatively. We can see here that for FHOFDM, as the number of radar nodes increases, average (over 1000 time slots) collisions increases in the network. Furthermore, as the number of neighbors increases (node degree), the collision situation gets worse. However, since CPARC makes contention-free links in the network, node scaling does not bring in more collisions. This shows the improved performance of CPARC over FH-OFDM, under the requirement of spectral covertness.

\subsection{CPARC spectrum utilization}

Figure 19 presents the efficient spectrum utilization property of CPARC. We define spectrum intensity to be the ratio of total number of subcarriers used in the network

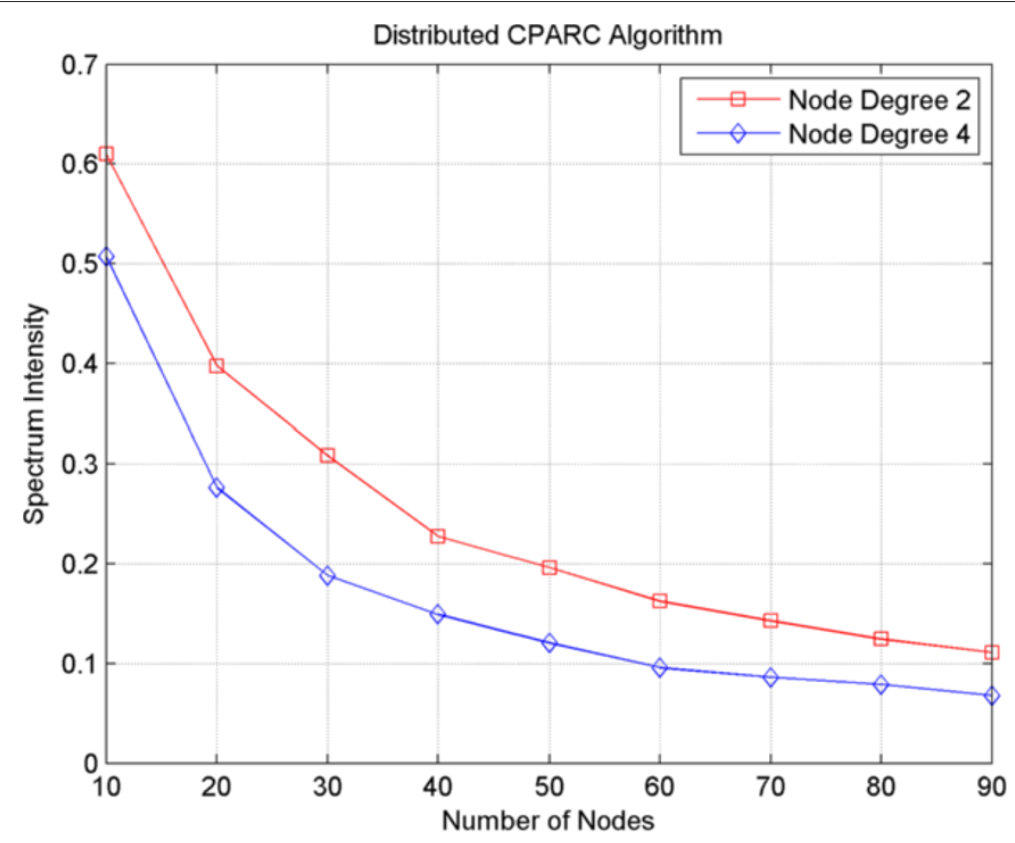

Figure 19 Subcarrier reuse leading to efficient spectrum utilization. 
to the total number of possible links present in the network. Good frequency reuse leads to lower spectrum intensity of the network, and hence, better spectrum utilization. In the square topology shown in Figure 12a, the number of subcarriers used by CPARC to produce a completely connected network was 8 , while the total number of links in the network was 24 , leading to a spectrum intensity of $33 \%$. This is further confirmed through Figure 19, where we observe that the spectrum intensity decreases with the number of the nodes in the network. We also observe that as the node degree increases, CPARC is better able to divide the subcarriers among the nodes providing improved spectrum performance in the overall system. Since the number of subcarriers between which the available bandwidth is to be divided is being minimized, it leads to optimal capacity. Furthermore, through Figure 20, we ascertain that the runtime performance retains its $O(\log n)$ growth rate, for both degrees 2 and 4 scenarios of the network.

\section{Conclusions}

This study targets the development of ad hoc radar networks as a solution to situations generally characterized by the absence of any central infrastructure, varying topological environments, and growing wireless security concerns. To address these needs, we have demonstrated that reliable multi-user data transmission/reception can occur simultaneously with high-resolution target detection/ranging, leading to cooperative spectrum coexistence of communications and radar. We formulated the AF of the multi-functional noise-OFDM waveform and have shown that up to $30 \%$ of an UWB signal can be utilized for data communications, without adversely affecting the primary function of the radar. Furthermore, utilizing this $30 \%$ bandwidth, we extended the point-to-point covert radar-communications platform to being a selforganized multi-hop cross-layered ad hoc radar network. This was achieved by developing a unique solution to the ad hoc OFDM multi-user multi-channel resource allocation problem. The salient features are: (i) it is a fully distributed, channel-diversity-aided multi-carrier resource allocation strategy, with its simplicity leading to significant practical relevance, (ii) it automatically adapts to network topology changes and channel variations, and has an logarithmic growth rate runtime performance, (iii) it provides a provable throughput guarantee that is at least competitive to other algorithms in the literature, and (iv) it inherently presents physical layer security, since CPARC uses OFDM subcarriers that are embedded within UWB noise and hence the transmitted wireless signal appears random and noise-like to unintended receivers.

Employing a routing layer about CPARC's contentionfree medium access layer will make this framework easily applicable to any mobile wireless platform. Towards this, and to exploit the multi-carrier covert PHY, we explore modifying the on-demand routing protocols designed for mobile ad hoc networks (MANETs). We envisage to bring forward a second level of cross-layer information flow, which will enhance the routing protocol's performance, especially security and scalability. Furthermore, promising and prospective candidates for implementing this system are software-defined radio techniques of software-based

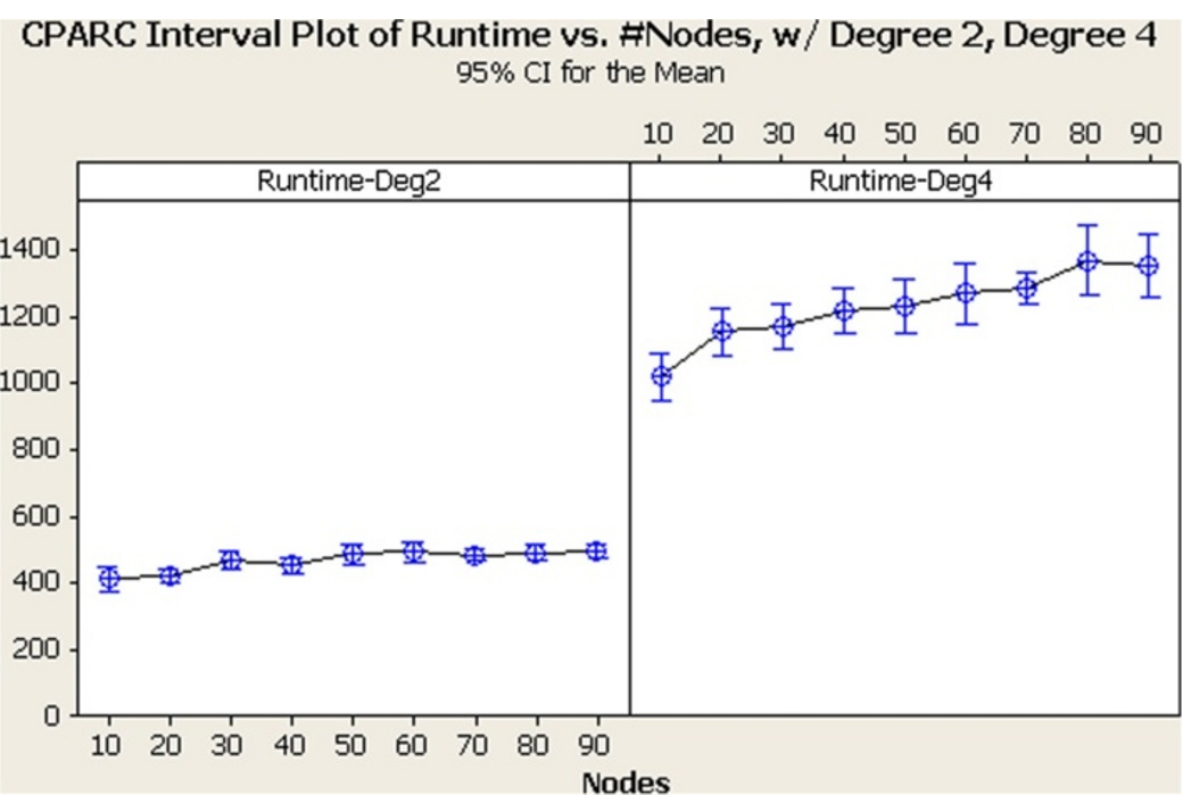

Figure 20 CPARC runtime performance for node degrees 2 and 4, 95\% confidence interval (CI). 
signal processing, hardware such as the universal software radio peripheral, and high-speed digital-to-analog converters. This will allow the system parameters to be software configurable, hence making an flexible framework for covert infrastructure-less surveillance networks.

\section{Appendix}

\section{AF derivation for the UWB noise-OFDM radar}

Consider the composite noise-OFDM signal reflected from a point target moving with constant velocity $v_{o}$ at an instantaneous range of $R=R_{o}-v_{o} t$, with resulting delay $\tau_{o}=2 R_{o} /\left(c-v_{o}\right) \cong 2 R_{o} / c$, and delay rate $\alpha=$ $2 v_{o} /\left(c-v_{o}\right) \cong 2 v_{o} / c$ such that $\tau(t)=\tau_{o}-2 v_{o} t / c=\tau_{o}-\alpha t$. The received target backscatter at the radar in response to the transmitted signal can be expressed as

$$
X_{r}(t)=k_{1} X\left((1+\alpha) t-\tau_{o}\right),
$$

where $k_{1}$ is the arbitrary amplitude constant representing target cross-section, antenna pattern, propagation factors, etc.

The transmitted signal is passed through a delay line with time delay equal to $\tau_{d}$ and frequency shifted using a local oscillator with frequency $f_{I F}$. The output therefore of the up-converter MXR1 of Figure 4 can be written as

$$
\begin{aligned}
X_{d}(t)= & \frac{1}{4}\left[u\left(t-\tau_{d}\right) \exp \left(j \theta_{1}\right)+u^{*}\left(t-\tau_{d}\right) \exp \left(-j \theta_{1}\right)\right] \\
& -\frac{1}{4}\left[v\left(t-\tau_{d}\right) \exp \left(j \theta_{2}\right)+v^{*}\left(t-\tau_{d}\right) \exp \left(-j \theta_{2}\right)\right] \\
& +\frac{1}{4}\left[s\left(t-\tau_{d}\right) \exp \left(j \theta_{2}\right)+s^{*}\left(t-\tau_{d}\right) \exp \left(-j \theta_{2}\right)\right],
\end{aligned}
$$

where $\theta_{1}=2 \pi\left[\left(f_{o}-f_{I F}\right) t-f_{o} \tau_{d}\right]$ and $\theta_{2}=2 \pi\left[\left(f_{n}-f_{I F}\right)\right.$ $\left.t-f_{n} \tau_{d}\right]$.

System noise, assumed to be AWGN, is distributed on the complex envelopes of the components of $X_{r}, X_{d}$ and is denoted by $\left|u_{i}, v_{i}, s_{i}\right|_{\{i=1,2\}}$. Now, passing these two signals $X_{r}$ and $X_{d}$ through correlator MXR2 of Figure 4, filtering through the IF BPF and dropping higher-order terms, the output signal can be shown to be:

$$
Z_{I F}=\frac{1}{2}\left\{\operatorname{Real}\left[A_{I F}+B_{I F}\right]\right\}
$$

where

$$
\begin{aligned}
A_{I F}= & \left(u_{1} u_{2}^{*}\right) \exp \left(-j 2 \pi\left(f_{I F} t+\alpha f_{o} t+f_{o}\left(\tau_{d}-\tau_{o}\right)\right)\right), \\
B_{I F}= & \left(v_{1} v_{2}^{*}+s_{1} s_{2}^{*}+v_{1} s_{2}^{*}+s_{1} v_{2}^{*}\right) \\
& \times \exp \left(-j 2 \pi\left(f_{I F} t+\alpha f_{n} t+f_{n}\left(\tau_{d}-\tau_{o}\right)\right)\right) .
\end{aligned}
$$

This $Z_{I F}$ is then passed through the I/Q integrating filters with impulse response $h(t)$. The filter impulse response $h(t)$ could be of any type; however, in this analysis, we assume a rectangular time window for the filter response of the form

$$
\begin{array}{r}
h(t)=1 / T, \quad 0 \leq t \leq T \\
0, \quad \text { otherwise. }
\end{array}
$$

To estimate $Z_{I F}$, we evaluate $u_{1} u_{2}^{*}, v_{1} v_{2}^{*}, s_{1} s_{2}^{*}, v_{1} s_{2}^{*}$, and $s_{1} v_{2}^{*}$ individually and take the expectation, which results in:

$$
\begin{aligned}
& E\left\{u_{1} u_{2}^{*}\right\}=\left\{R_{c c}(\alpha t-\tau)-j R_{c s}(\alpha t-\tau)\right\}, \\
& E\left\{v_{1} v_{2}^{*}\right\}=\left\{R_{c c_{n}}(\alpha t-\tau)-j R_{c s_{n}}(\alpha t-\tau)\right\}, \\
& E\left\{v_{1} s_{2}^{*}\right\}=E\left\{s_{1} v_{2}^{*}\right\}=0,
\end{aligned}
$$

where

$$
R_{c c}(.)=\frac{\sin [\pi \beta(\alpha t-\tau)]}{\pi \beta(\alpha t-\tau)},
$$

and

$$
R_{c c_{n}}(.)=\frac{\sin \left[\pi \beta_{n}(\alpha t-\tau)\right]}{\pi \beta_{n}(\alpha t-\tau)} .
$$

For evaluating $s_{1} s_{2}^{*}$, we make a reasonable assumption that identical data are transmitted over all sub-carriers of the OFDM symbol. We then use the orthogonality principle for OFDM carriers and Equation (3) together, to finally show that $E\left\{s_{1} s_{2}^{*}\right\}=N T_{s}$, where $T_{s}$ is the symbol period.

Neglecting the phase terms, the expected values of the $I$ and $Q$ outputs of the $I / Q$ integrating filters can therefore be symbolized as $\left\langle Z_{I}(\alpha, \tau, t)\right\rangle$ and $\left\langle Z_{Q}(\alpha, \tau, t)\right\rangle$.

Since the envelope of the output is defined to be the RMS sum of these two, the AF is of the form

$$
|\langle\chi(\alpha, \tau, t)\rangle|=\left|\left\langle Z_{I}\right\rangle^{2}+\left\langle Z_{Q}\right\rangle^{2}\right|^{1 / 2} .
$$

Similar to [23], we go through the algebra of finding $\left\langle Z_{I}\right\rangle$ and $\left\langle Z_{Q}\right\rangle$, squaring and summing them, and use trigonometric identities to solve for Equation (21). Ignoring the cross-correlation component due to the UWB noise as well as the notch spectrum (which is very low, hence negligible for a more uniform transmit PSD), Equation (21) then reduces to the following simplified expression for the AF of a UWB noise-OFDM radar:

$$
|\langle\chi(\alpha, \tau, t)\rangle|=|(A+B) \otimes h(t)|,
$$

where

$$
A=R_{c c}(\alpha t-\tau) \exp \left(-j 2 \pi \alpha f_{o} t\right),
$$

and

$$
B=\left(R_{c c_{n}}(\alpha t-\tau)+N T_{s}\right) \exp \left(-j 2 \pi \alpha f_{n} t\right) .
$$


Pseudo-code for CPARC algorithm Algorithm 1 CPARC(isc; osc; msg)

Require: Feasible Set, subcarriers available per radar isc, system overall subcarriers $o s c$, incoming message msg.

Ensure: Local subcarrier allocation

1:

$\left(\text { radar }_{\text {self }}, \text { radar }_{\text {neighbor }}\right)_{\text {subcarrier }}$.

1: while $m s g$ do

2:

4:

5:

7:

8:

9:

10:

11:

12:

13:

14:

15:

16:

17:

18:

19:

20:

21:

22:

23:

24:

25:

26:

27:

28:

29:

30:

31:

32:

33:

34:

$35:$

36:

37:

38:

39:

40:

41: if $m s g . I D=$ HELLO then

if $m s g . s r c \notin N_{l}$ and

hostID $<$ msg.src then

end if add $m s g$.src to $N_{l}$

if $m s g . s r c \notin L A I$ and $G A I$.isc $>0$

then

end if

select $S C$ from isc and send PROPOSAL to msg.src

else if $m s g . I D=I N F O$ then

$G A I=$ msg.GAI

update $L A I$ from new $G A I$ when

$L A I \notin G A I$

else if $m s g . I D=$ PROPOSAL then

look into $F S$ and select $S C$ with

$\max$. PSD

if $m s g . s c \in L A I$ with same

(src, dest) pair then send ACCEPT to msg.src else if $m s g . s c \in L A I$ on different src or dest then send REJECT to msg.src

else

if $G A I . i s c>0$ then select $s c$ and send end if ACCEPT to msg.src

end if

else if $m s g . I D=$ ACCEPT then

if $m s g . s c \in L A I$ on some

(src, dest) $\neq$ (msg.src, $m s g$. dest $)$

then

else

send REJECT to $m s g . s r c$

pair self with msg.src,

end if

update $L A I, G A I$

else if $m s g I D=$ CONFIRM then

if $m s g$.dest $=$ self then

update $L A I, G A I$ and send

else

INFO broadcast

update $G A I$ on neighbor

end if

information

else if $m s g . I D=$ REJECT then

temporarily unmark rejected $S C$

from $G A I$

if $G A I . i s c>0$ then

select $S C$ and send

end if

PROPOSAL to msg.src

else if $m s g . I D=$ OFFLINE then

if $m s g . s r c \in L A I$ and

$L A I . s r c=$ msg.src then
42:

43:

44:

45:

nd while

46: if any $N_{l} \notin L A I$ and $G A I . i s c>0$ then

47:

48: end if

select $S C$ and send PROPOSAL to it

Competing interests

The authors declare that they have no competing interests.

\section{Author details}

${ }^{1}$ Department of Computer Science and Engineering, The Pennsylvania State University, University Park, PA 16802, USA. 2Department of Electrical

Engineering, The Pennsylvania State University, University Park, PA 16802, USA

Received: 23 May 2011 Accepted: 26 November 2012

Published: 4 January 2013

\section{References}

1. A Mehbodniya, S Aissa, in Proceedings of the GLOBECOM 07. Coexistence between DS-UWB and MB-OFDM: Analysis and interference mitigation, Washington, DC, USA, November 2007), doi:10.1109/GLOCOM.2007.986

2. L Ding, T Melodia, SN Batalama, JD Matyjas, in Proceedings of the IEEE SECON, vol. 2010. Distributed routing, relay selection, and spectrum allocation in cognitive and cooperative ad hoc networks, Boston, MA, USA, June 2010), doi:10.1109/SECON.2010.5508267

3. H Li, Y Cheng, C Zhou, P Wan, in Proceedings of the 30th International Conference on Distributed Computing Systems (ICDCS). Multi-dimensional conflict graph based computing for optimal capacity in MR-MC wireless networks (Genoa, Italy, June 2010), doi:10.1109/ICDCS.2010.58

4. W Galuba, P Papadimitratos, M Poturalski, K Aberer, Z Despotovic, Kellerer W, in Proceedings of the IEEE INFOCOM, vol. 2010. CASTOR scalable secure routing for ad-hoc networks, San Diego, CA, USA, March 2010), doi:10.1109/INFCOM.2010.5462115

5. C Gentile, A Kik, in Proceedings of the GLOBECOM, vol. 06. WLC28-4: an evaluation of ultra wideband technology for indoor ranging (San Francisco, CA, USA, December 2006), doi:10.1109/GLOCOM.2006.778

6. J Bellorado, SS Ghassemzadeh, LJ Greenstein, T Sveinsson, V Tarokh, in Proceedings of the GLOBECOM 03. Coexistence of ultra-wideband systems with IEEE-802.11 a wireless LANs (San Francisco, CA, USA, December 2003), doi:10.1109/GLOCOM.2003.1258271

7. SC Surender, RM Narayanan, UWB noise-OFDM netted radar: physical layer design and analysis. IEEE Trans. Aerospace Electron. Syst. 47(2), 1380-1400 (2011)

8. P Bharadwaj, P Runkle, L Carin, JA Berrie, JA Hughes, Multiaspect classification of airborne targets via physics-based HMMs and matching pursuits. IEEE Trans. Aerospace Electron. Syst. 37(2), 595-606 (2001)

9. Z Li, S Papson, RM Narayanan, Data level fusion of multi-look inverse synthetic aperture radar (ISAR) images. IEEE Trans. Geosci. Remote Sens. 46(5), 1394-1406 (2008)

10. D Koutsonikolas, C Wang, YC Hu, in Proceedings of the IEEE INFOCOM 2010. CCACK: efficient network coding based opportunistic routing through cumulative coded acknowledgments, San Diego, CA, USA, March 2010), doi:10.1109/INFCOM.2010.5462125

11. KJ Quirk, M Srinivasan, in Proceedings of the GLOBECOM 09. An MSK waveform for radar applications, Honolulu, HI, USA, December 2009), doi:10.1109/GLOCOM.2009.5425647

12. P Dutta, S Jaiswal, D Panigrahi, R Rastogi, in Proceedings of the 27th IEEE Conference on Computer Communications, INFOCOM. A new channel assignment mechanism for rural wireless mesh networks, Phoenix, AZ, USA, April 2008), doi:10.1109/INFOCOM.2008.294

13. X Lin, S Rasool, in Proceedings of the 26th IEEE INFOCOM 2007. A distributed joint channel-assignment scheduling and routing algorithm for multi-channel ad hoc wireless networks, Anchorage, AK, USA, May 2007), doi:10.1109/INFCOM.2007.134

14. CJ Baker, AL Hume, Netted radar sensing. IEEE Aerospace Electron. Syst. Mag. 3(2), 3-6 (2003) 
15. D Garmatyuk, J Schuerger, YT Morton, K Binns, M Durbin, J Kimani, in Proceedings of the European Radar Conference (EURAD 2007). Feasibility study of a multi-carrier dual-use imaging radar and communication system, Munich, Germany, December 2007), doi:10.1109/EURAD.2007.4404970

16. GN Saddik, RS Singh, ER Brown, Ultra-wideband multifunctional communications/radar system. IEEE Trans. Microwave Theory Techn. 55(7), 1431-1437 (2007)

17. A Gupta, P Mohapatra, A survey on ultrawideband medium access control schemes. Comput. Netw. 51(11), 2976-2993 (2007)

18. Y Yu, GB Giannakis, in Proceedings of the IEEE INFOCOM 2007. Joint congestion control and OFDMA scheduling for hybrid wireline-wireless networks, Anchorage, AK, USA, May 2007), doi:10.1109/INFCOM.2007.118

19. Y Hua, Q Zhang, Z Niu, in Proceedings of the IEEE INFOCOM 2010. Resource allocation in multi-cell OFDMA-based relay networks, San Diego, CA, USA, March 2010), doi:10.1109/INFCOM.2010.5462037

20. S Gao, L Qian, D Vaman, Distributed energy efficient spectrum access in cognitive radio wireless ad hoc networks. IEEE Trans. Wirel. Commun. 8(10), 5202-5213 (2009)

21. XWu, R Srikant, in Proceedings of the IEEE CDC. Regulated maximal matching: a distributed scheduling algorithm for multi-hop wireless networks with node exclusive spectrum sharing, Seville, Spain, December 2005), doi:10.1109/CDC.2005.1583011

22. Y Li, Orthogonal Frequency Division Multiplexing for Wireless Communications. (Springer, New York, NY, 2006)

23. M Dawood, RM Narayanan, Generalised wideband ambiguity function of a coherent ultrawideband random noise radar. IEE Proc. Radar Sonar Navigat. 150(5), 379-386 (2003)

24. SC Surender, RM Narayanan, CR Das, in Proceedings of the 2010 IEEE GLOBECOM. Performance analysis of communications \& radar coexistence in a covert UWB OSA system, Miami, FL, USA, December 2010), doi:10.1109/GLOCOM.2010.5683837

25. L Tassiulas, in Proceedings of the IEEE INFOCOM 1998. Linear complexity algorithms for maximum throughput in radio networks and input queued switches, San Francisco, CA, USA, March 1998), doi:10.1109/INFCOM.1998.665071

26. P Chaporkar, K Kar, S Sarkar, in Proceedings of the 43rd Annual Allerton Conference on Communication, Control and Computing. Throughput guarantees in maximal scheduling in wireless networks, Monticello, IL, USA, September 2005), pp. 28-30

27. OMNET++ Integrated Development Environment. http://omnetpp.org/

28. RM Narayanan, in Proceedings of the 14th AFOSR Electromagnetics Workshop, San Antonio. ultra-wideband, Radar imaging using spectrally fragmented (UWB) noise waveforms, TX, USA, January 2003), pp. P4-P5

29. SS Skiena, Algorithm Design Manual. (Springer, London, UK, 2008)

30. Q Shi, Z Yang, L He, K Peng, in Proceedings of the 11th IEEE ICCS. All digital baseband frequency hopping OFDM system, Guangzhou, China, November 2008), doi:10.1109/ICCS.2008.4737268

doi:10.1186/1687-1499-2013-4

Cite this article as: Surender et al:: Cross-layered resource allocation in UWB noise-OFDM-based ad hoc surveillance networks. EURASIP Journal on Wireless Communications and Networking 2013 2013:4.

\section{Submit your manuscript to a SpringerOpen ${ }^{\circ}$ journal and benefit from:}

- Convenient online submission

- Rigorous peer review

- Immediate publication on acceptance

- Open access: articles freely available online

- High visibility within the field

- Retaining the copyright to your article

Submit your next manuscript at $\boldsymbol{\wedge}$ springeropen.com 